\title{
Linear Phase Cosine Modulated Maximally Decimated Filter Banks with Perfect Reconstruction
}

\author{
Yuan-Pei Lin, Student Member, IEEE, and P. P. Vaidyanathan, Fellow, IEEE
}

\begin{abstract}
We propose a novel way to design maximally decimated FIR cosine modulated filter banks, in which each analysis and synthesis filter has linear phase. The system can be designed to have either the approximate reconstruction property (pseudoQMF system) or perfect reconstruction property (PR system). In the PR case, the system is a paraunitary filter bank. As in earlier work on cosine modulated systems, all the analysis filters come from a FIR prototype filter. However, unlike in any of the previous designs, all but two of the analysis filters have a total bandwidth of $2 \pi / M$ rather than $\pi / M$ (where $2 M$ is the number of channels in our notation). A simple interpretation is possible in terms of the complex (hypothetical) analytic signal corresponding to each bandpass subband.

The coding gain of the new system is comparable with that of a traditional $M$-channel system (rather than a $2 M$-channel system). This is primarily because there are typically two bandpass filters with the same passband support. Correspondingly, the cost of the system (in terms of complexity of implementation) is also comparable with that of an $M$-channel system. We also demonstrate that very good attenuation characteristics can be obtained with the new system.
\end{abstract}

\section{INTRODUCTION}

$\mathbf{T}$ HE $M$-channel maximally decimated cosine modulated filter bank shown in Fig. 1 has been studied extensively in [1]-[19]. When the system in Fig. 1 is alias free, it is an LTI system with transfer function $T(z)$, as indicated in Fig. 1. $T(z)$ will be called the distortion function or the overall response in the following discussion.

The system in Fig. 1 is said to be a cosine modulated filter bank if all analysis and synthesis filters are generated by cosine or sine modulation of one or two prototype filters. Cosine modulated filter banks [5]-[15] are well known for their design cost saving and implementation saving. Two types of cosine modulated filter banks have been developed: pseudo-QMF systems [5]-[9] and perfect reconstruction systems [10]-[15]. Unlike a PR system, a pseudo-QMF filter bank is only approximately alias free and has approximate reconstruction property (and the approximation improves with filter order).

\section{A. Previous Work}

In [7], Chu mentioned three approaches for designing cosine modulated filter banks with approximate aliasing cancellation. The first one involves designing two prototype filters. The

\footnotetext{
Manuscript received November 26, 1993; revised April 19, 1995. The associate editor coordinating the review of this paper and approving it for publication was Prof. Roberto H. Bamberger. This work was supported by NSF Grant MIP 92-15785, Tektronix, Inc. and by Rockwell International.

The authors are with the Department of Electrical Engineering, California Institute of Technology, Pasadena, CA 91125 USA.
}

IEEE Log Number 9415088.

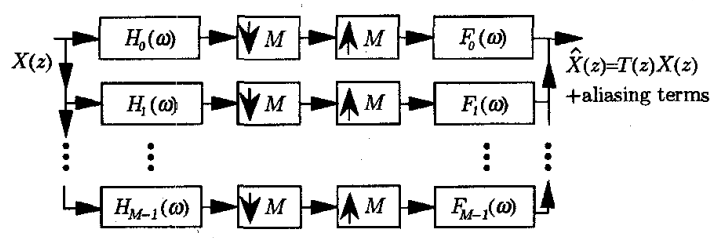

Fig. 1. $M$ channel maximally decimated filter bank.

implementation cost for the analysis bank is that of two prototype filters plus cosine and sine modulation. The second method, similar to the one proposed earlier by Rothweiler [6], requires only one prototype filter. Its distortion $T(z)$ has linear phase and approximately flat magnitude response, but individual analysis and synthesis filters do not have linear phase, which is important for image coding applications. The third method, given in [7], needs also only one prototype filter. With this method all the analysis and synthesis filters have linear phase, but the resulting $\left|T\left(e^{j \omega}\right)\right|$ has a peak or a null at zero frequency and at $\pi$.

Recently, some cosine modulated maximally decimated systems with perfect reconstruction property have been proposed [10]-[15]. In [10], the individual filters in the filter bank have linear phase but the length of the prototype can not be larger than the number of channels. Although the length of the prototype in [11]-[15] is not as restricted, the individual analysis and synthesis filters do not have linear phase even if the prototypes have linear phase. In [16], some techniques for characterizing and designing paraunitary linear phase filter banks have been developed, but these are not cosine modulated.

In general, the following results are typically desired in a filter bank:

1) Cancellation of aliasing errors: Exact or approximate alias cancelation is desired.

2) Distortion function: $T(z)$ is exactly or nearly a delay. In particular the magnitude response $\left|T\left(e^{j \omega}\right)\right|$ is required to be flat.

3) Cosine modulation: All filters must be cosine modulated versions of a prototype. In this case, only the design of the prototype filter is needed. Besides the implementation cost is only that of the prototype filter plus one DCT matrix working at a decimated rate. For instance, in an $M$ channel maximally decimated filter bank, the DCT matrix computation is performed after $M$ fold decimators. Design cost and implementation cost are significantly reduced. 


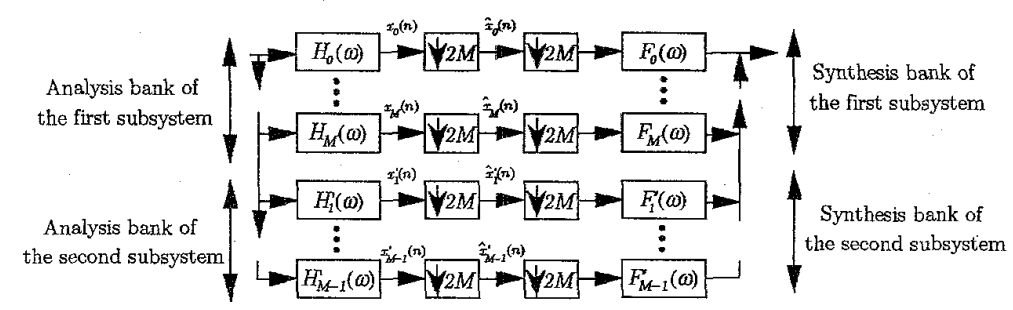

Fig. 2. New setup for derivation of the cosine modulated maximally decimated filter bank.

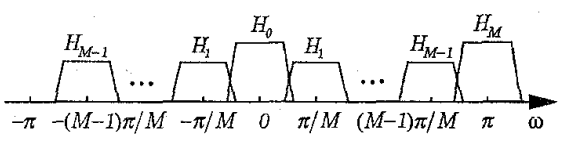

(a)

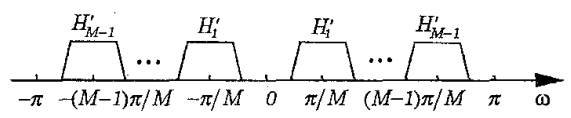

(b)

Fig. 3. Magnitude responses of the cosine modulated analysis bank filters: (a) First subsystem; (b) second subsystem.

4) Linear phase property of analysis and synthesis filters: These are desired in image coding applications, when the subbands are heavily quantized. (The nonlinearity of phase of individual filters leads to some artifacts in the reconstructed image.)

5) Filter length: If the filters are restricted to be short, they can not have very good attenuation. So the length of the filters should be allow to be large.

In this paper, we will show how to achieve all of these properties. 1

\section{B. The New System}

We propose a novel way to design a cosine modulated filter bank with perfect reconstruction or approximate reconstruction. The set up of this new filter bank is shown in Fig. 2. It has $2 M$ channels and is maximally decimated. Every analysis and synthesis filter in this system is some cosine modulated version of the same prototype filter. In the nearly PR case, the new filter bank can be designed to be almost alias free. Its overall response, $T(z)$, has approximately flat magnitude response and linear phase. In the PR case aliasing is cancelled exactly and $T(z)$ is merely a delay. In both systems, every analysis and synthesis filter has linear phase property.

We would like to regard this filter bank as a connection of two subsystems. The first subsystem has $M+1$ channels and the second subsystem has $M-1$ channels. Fig. 3(a) and (b) show, respectively, the magnitude response sketches of analysis filters in the two subsystems. Notice that the second subsystem does not have filters with pass-bands covering zero frequency or $\pi$ while the first subsystem does. The synthesis filters are time-reversed versions of analysis filters, and therefore have identical magnitude responses.

\footnotetext{
${ }^{1}$ While this paper was under preparation, we learnt from Dr. Fliege of Hamburg University that he has developed similar results [18].
}

In a conventional $N$ channel maximally decimated cosine modulated PR or approximately PR system, all filters have the same total bandwidth $2 \pi / N$ (including positive and negative frequency) and the same height in passband. Their pass-bands do not overlap significantly. When the subband signals are decimated by $N$, there is no severe aliasing. Aliasing is caused only by the nonideal nature of the bandpass filters, which have a finite stopband attenuation and nonzero transition bandwidth.

The new system, however, is unusual. As shown in Fig. 3(a), $H_{k}(z)$ and $H_{k}^{\prime}(z)$ have the same spectral supports and total bandwidth $2 \pi / M$, i.e., two times wider than they are in the traditional case while $H_{0}(z)$ and $H_{M}(z)$ have total bandwidth only $\pi / M$. Also $H_{0}(z)$ and $H_{M}(z)$ have $\sqrt{2}$ times the height of other filters. In each channel serious aliasing occurs. However, since there is spectral overlapping of the filters in the first and second subsystems, we are able to cancel this aliasing. Cancellation of these aliasing components is possible by judiciously choosing the parameters. The supports of the analysis filters are similar to those given in [10]. In addition, perfect reconstruction is possible with this scheme by imposing certain conditions on polyphase components of the prototype filter.

Furthermore, in conventional $N$ channel filter banks, each subband signal represents the input signal in that particular subband. In the presence of quantizers in the subbands, bits are allocated based on subband energy. We will explain in Section $V$ that the $2 M$-channel cosine modulated filter bank can be interpreted as a modified DFT filter bank. As a result, the subband signals retain the usual meaning and can still be quantized in the usual manner although some filters in the new system have twice the bandwidth of a filter in a typical $M$-channel filter bank. Detailed discussion is given in Section V. An image coding example is included to demonstrate the usefulness of the system.

Although this is a $2 M$ channel system; we will show that design cost and implementation cost are equivalent to that of a conventional $M$ channel maximally decimated cosine modulated filter bank. The coding gain performance is also close to that of an $M$ channel system.

\section{Paper Outline and Notations}

This paper is organized as follows: In Section II, we introduce the new maximally decimated linear phase cosine modulated filter bank with approximate reconstruction property. The prototype filter is further constrained in Section III to achieve perfect reconstruction. Necessary and sufficient conditions for PR with this scheme will be given therein. 
Efficient implementation of this new filter bank is presented in Section IV. Coding gain and optimal bit allocation of the new system will be discussed in Section V. Numerical examples and tables of prototype filter coefficients are given in Section VI.

\section{Notations:}

1) Boldfaced quantities are used to represent matrices.

2) The notations $\mathbf{A}^{T}, \mathbf{A}^{*}$, and $\mathbf{A}^{\dagger}$ represent the transpose, conjugate, and transpose-conjugate of $\mathbf{A}$. The 'tilde' notation is defined as follows: $\tilde{\mathbf{A}}(z)=\mathbf{A}^{\dagger}\left(1 / z^{*}\right)$.

3) Matrix $\mathbf{I}_{k}$ denotes a $k \times k$ identity matrix and $\mathbf{J}_{k}$ denotes a $k \times k$ reversal matrix with

$$
\mathbf{J}_{k}=\left(\begin{array}{cccc}
0 & \ldots & 0 & 1 \\
0 & \ldots & 1 & 0 \\
\vdots & & \vdots & \vdots \\
1 & \ldots & 0 & 0
\end{array}\right)
$$

4) The delay chain $\mathrm{e}(z)$ is the vector

$$
\mathbf{e}(z)=\left[1 z^{-1} \ldots z^{-(M-1)}\right]^{T} .
$$

5) The unit-pulse, denoted as $\delta(n)$, is defined according to

$$
\delta(n)= \begin{cases}1 & n=0 \\ 0 & \text { otherwise }\end{cases}
$$

6) The value of the function, $\lceil x\rceil$, is the smallest integer greater or equal to $x$ and the value of the function, $\lfloor x\rfloor$, is the largest integer less or equal to $x$.

\section{Linear Phase Cosine Modulated Filter BANKS WITH APPROXIMATE RECONSTRUCTION}

In this section, we introduce a new maximally decimated linear phase cosine modulated filter bank with approximate reconstruction property. The system is nearly alias free. Aliasing errors decrease as the stopband attenuation of the prototype increases. The distortion function $T(z)$ has linear phase and approximately flat magnitude response. Every analysis and synthesis filter comes from modulation of the same prototype filter. Furthermore, all of them have linear phase.

Consider the $2 M$ channel maximally decimated filter bank shown in Fig. 2. Suppose prototype filter $P_{0}(z)$ is of order $N$ and linear-phase. Let

$$
U_{k}(z)=P_{0}\left(z W_{2 M}^{k}\right)
$$

where $W_{2 M}^{k}=e^{-j k \pi / M}$. In all equations to follow unsubscripted $W$ stands for $W_{2 M}$ unless otherwise indicated. Magnitude responses of $P_{0}(z)$ and $U_{k}(z)$ are shown in Fig. 4. As we can observe from Fig. $4,\left|U_{k}\left(e^{j \omega}\right)\right|$ is a shift of $\left|P_{0}\left(e^{j \omega}\right)\right|$ by $k \pi / M$. For $H_{k}(z)$ to be a cosine modulation of $P_{0}(z)$, we can choose

$$
H_{k}(z)=a_{k} U_{k}(z)+a_{k}^{*} U_{-k}(z)
$$

for some $a_{k}$. Taking a hint from paraunitary perfect reconstruction filter banks, let us constrain

$$
F_{k}(z)=z^{-i_{0}} \tilde{H}_{k}(z), \quad F_{k}^{\prime}(z)=z^{-i_{0}} \tilde{H}_{k}^{\prime}(z)
$$

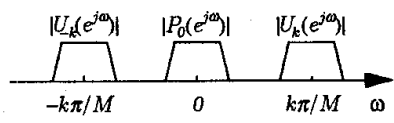

Fig. 4. Magnitude responses of $P_{0}(z)$ and $U_{k}(z)$.

for some $i_{0}$. We will show that the choice

$$
\begin{gathered}
H_{k}^{\prime}(z)=z^{-M}\left(\left(a_{k}^{\prime} U_{k}(z)+a_{k}^{\prime *} U_{-k}(z)\right),\right. \\
k=1,2, \ldots, M-1
\end{gathered}
$$

for the second set of filters will yield approximate reconstruction for appropriate choice of $a_{k}^{\prime}$ to be determined later. To keep $F_{k}^{\prime}(z)$ causal, we will take $i_{0}=M+N$ in (2).

Summarizing, the filters to be considered in our system will have the form

$$
\begin{aligned}
H_{k}(z)= & a_{k} U_{k}(z)+a_{k}^{*} U_{-k}(z), \quad k=0,1, \ldots, M, \\
H_{k}^{\prime}(z)= & z^{-M}\left(a_{k}^{\prime} U_{k}(z)+a_{k}^{\prime *} U_{-k}(z)\right), \\
& \quad k=1,2, \ldots, M-1, \\
F_{k}(z)= & z^{-(N+M)} \tilde{H}_{k}(z), \quad k=0,1, \ldots, M \\
F_{k}^{\prime}(z)= & z^{-(N+M)} \tilde{H}_{k}^{\prime}(z), \quad k=0,1, \ldots, M-1 .
\end{aligned}
$$

We now show that with proper design of $P_{0}(z)$ and appropriate choices of $a_{k}, a_{k}^{\prime}$, this filter bank has the following four properties: 1) $\left|T\left(e^{j \omega}\right)\right|$ is approximately flat, 2) $T(z)$ has linear phase, 3 ) the system is nearly alias free, and 4) every analysis and synthesis filter has linear phase.

1) Flatness of $\left|T\left(e^{j \omega}\right)\right|$ : From [17], we know that the distortion function $T(z)$ of the $2 M$ channel system in Fig. 2 can be expressed as

$$
T(z)=\frac{1}{2 M}\left(\sum_{k=0}^{M} H_{k}(z) F_{k}(z)+\sum_{k=1}^{M-1} H_{k}^{\prime}(z) F_{k}^{\prime}(z)\right) .
$$

Assume that nonadjacent bands of $U_{k}(z)$ do not overlap, i.e.

$$
\left|U_{k}\left(e^{j \omega}\right) U_{k}\left(e^{j \omega} W^{i}\right)\right| \approx 0, \quad i=2, \ldots, 2 M-2 .
$$

This assumption is reasonable if $P_{0}(z)$ has stopband edge $\omega_{s}<\frac{\pi}{M}$ and large enough stopband attenuation. In this case the distortion function becomes

$$
\begin{aligned}
T\left(e^{j \omega}\right) \approx & \frac{z^{-(N+M)}}{2 M} \\
& \times\left(4\left|a_{0}\right|^{2}\left|U_{0}\left(e^{j \omega}\right)\right|^{2}+4\left|a_{M}\right|^{2}\left|U_{M}\left(e^{j \omega}\right)\right|^{2}\right. \\
& \left.+\sum_{k=1}^{M-1}\left(\left|a_{k}\right|^{2}+\left|a_{k}^{\prime}\right|^{2}\right)\left(\left|U_{k}\left(e^{j \omega}\right)\right|^{2}+\left|U_{-k}\left(e^{j \omega}\right)\right|^{2}\right)\right) .
\end{aligned}
$$

If we choose $\left|a_{k}\right|=\left|a_{k}^{\prime}\right|=1$, for $k=1, \ldots, M-1$ and

$$
a_{0}=a_{M}=\frac{1}{\sqrt{2}}
$$

then we have

$$
T\left(e^{j \omega}\right) \approx \frac{z^{-(N+M)}}{M} \sum_{k=0}^{2 M-1}\left|U_{k}\left(e^{j \omega}\right)\right|^{2}
$$

which can be designed to be nearly flat by optimizing over the filter coefficients of $P_{0}(z)$ [17]. 
2) Linear Phase Property of $T(z)$ : Using our constraint that the synthesis filters are time-reversed versions of the corresponding analysis filters, the distortion function assumes the form

$$
\begin{aligned}
T\left(e^{j \omega}\right)= & e^{-j \omega(N+M)} \frac{1}{2 M} \\
& \times\left(\sum_{k=0}^{M}\left|H_{k}\left(e^{j \omega}\right)\right|^{2}+\sum_{k=1}^{M-1}\left|H_{k}^{\prime}\left(e^{j \omega}\right)\right|^{2}\right)
\end{aligned}
$$

which shows that $T(z)$ has linear phase.

3) Approximate Alias Cancellation: A $2 M$ channel maximally decimated filter bank as in Fig. 2 is alias free if all alias transfer functions are zero. The alias transfer function of the $i$ th alias component as defined in [17] is

$A_{i}(z)=\frac{1}{2 M}\left(\sum_{k=0}^{M} H_{k}\left(z W^{i}\right) F_{k}(z)+\sum_{k=1}^{M-1} H_{k}^{\prime}\left(z W^{i}\right) F_{k}^{\prime}(z)\right)$

We say that the system in Fig. 2 is alias free if $A_{i}(z)=0$, for $i=1,2, \ldots, 2 M-1$. With the choice of analysis and synthesis filters in (3), we have

$$
\begin{aligned}
A_{i}(z)=\frac{z^{-(N+M)}}{2 M}\left(2 U_{0}\left(z W^{i}\right) \tilde{U}_{0}(z)+2 U_{M}\left(z W^{i}\right) \tilde{U}_{M}(z)\right. \\
\left.+A_{i}^{(1)}(z)+A_{i}^{(2)}(z)+A_{i}^{(3)}(z)+A_{i}^{(4)}(z)\right)
\end{aligned}
$$

where

$$
\begin{aligned}
& A_{i}^{(1)}(z)=\sum_{k=1}^{M-1}\left(1+W^{-M i}\right) U_{k}\left(z W^{i}\right) \tilde{U}_{k}(z) \\
& A_{i}^{(2)}(z)=\sum_{k=1}^{M-1}\left(a_{k}^{* 2}+a_{k}^{*^{2}} W^{-M i}\right) U_{-k}\left(z W^{i}\right) \tilde{U}_{k}(z) \\
& A_{i}^{(3)}(z)=\sum_{k=1}^{M-1}\left(a_{k}^{2}+{a_{k}^{\prime}}^{2} W^{-M i}\right) U_{k}\left(z W^{i}\right) \tilde{U}_{-k}(z) \\
& A_{i}^{(4)}(z)=\sum_{k=1}^{M-1}\left(1+W^{-M i}\right) U_{-k}\left(z W^{i}\right) \tilde{U}_{-k}(z) .
\end{aligned}
$$

By appropriate choice of $a_{0}$ and $a_{M}$ and some relation between $N$ and $M$, we will ensure that $A_{i}(z) \approx 0$. More specifically, if we choose

and

$$
a_{k}=1, a_{k}^{\prime}=-\sqrt{-1}, \quad k=1,2, \ldots, M-1
$$

$$
N=\left(2 m_{0}+1\right) M \quad \text { for arbitrary integer } m_{0}
$$

and further constrain $a_{0}$ and $a_{M}$ as in (6), then we can verify that $A_{i}(z) \approx 0$ (to be verified in Appendix A). The above values of $a_{k}$ and $a_{k}^{\prime}$ are chosen for simplicity. A more generalized formula can be derived. However, the more generalized expressions for $a_{k}$ and $a_{k}^{\prime}$ do not provide more flexibility in the design of the prototype. They will not be discussed.
4) Linear Phase Property of Individual Analysis and Synthesis Filters: Having determined the parameters $a_{k}$ and $a_{k}^{\prime}$, we can write the impulse responses of the analysis and synthesis filters

$$
\begin{aligned}
h_{k}(n)= & \sqrt{2} p_{0}(n) \cos \left(\frac{\pi}{M} k n\right), \quad k=0 \text { or } M, \\
h_{k}(n)= & 2 p_{0}(n) \cos \left(\frac{\pi}{M} k n\right), \quad k=1, \ldots, M-1, \\
h_{k}^{\prime}(n)= & 2 p_{0}(n-M) \sin \left(\frac{\pi}{M} k(n-M)\right), \\
& \quad k=1, \ldots, M-1, \\
f_{k}(n)= & h_{k}(N+M-n), \quad k=0, \ldots, M, \\
f_{k}^{\prime}(n)= & h_{k}^{\prime}(N+M-n), \quad k=1, \ldots, M-1 .
\end{aligned}
$$

By using the linear phase property of $P_{0}(z)$ and the constraint $N=\left(2 m_{0}+1\right) M$ we can verify that every filter above has linear phase. The number of symmetric filters and antisymmetric filters agrees with the result from [16]; in a $2 M$ channel linearphase paraunitary filter bank, $M$ analysis filters are required to be symmetric and the remaining $M$ filters are antisymmetric.

5) Relation to a Paraunitary System: Having decided the values of $a_{k}$ and $a_{k}^{\prime}$ in (3), we can obtain symbolic magnitude reponses of $H_{k}(z)$ and $H_{k}^{\prime}(z)$ as in Fig. 3. In our formulation, the synthesis filters are constrained to be the time-reversed versions of corresponding analysis filters. Consequently, if the system in Fig. 2 is PR, then it is a paraunitary system. In a paraunitary system, every filter has the same engergy, i.e., $\sum_{n}\left|f_{k}(n)\right|^{2}$ is a constant for all $k$. However, the total bandwidth of $H_{0}(z)$ and $H_{M}(z)$ are only half that of all other filters. In order to have about the same energy as the other filters, $\left|H_{0}\left(e^{j \omega}\right)\right|$ and $\left|H_{M}\left(e^{j \omega}\right)\right|$ have $\sqrt{2}$ times the heights of other filters. In the derivation of properties (1) and (3), we need $H_{0}(z)$ and $H_{M}(z)$ to have $\sqrt{2}$ times the heights of other filters for aliasing cancellation and flat $\left|T\left(e^{j \omega}\right)\right|$. This is consistent with the equal engery property of filters in paraunitary systems.

It can be verified that when the prototype filter is an ideal brick wall filter, the system in Fig. 2 is indeed a PR system.

6) Design Cost: An objective function reflecting the nonflatness of $\left|T\left(e^{j \omega}\right)\right|$ in (7) and the stop attenuation of $P_{0}(z)$ is [17]

$$
\begin{aligned}
\phi= & \alpha \int_{0}^{\pi / M}\left(\left|P_{0}\left(e^{j \omega}\right)\right|^{2}+\left|P_{0}\left(e^{j\left(\omega-\frac{\pi}{M}\right)}\right)\right|^{2}-1\right)^{2} d \omega \\
& +(1-\alpha) \int_{(\pi / 2 M)+\epsilon}^{\pi}\left|P_{0}\left(e^{j \omega}\right)\right|^{2} d \omega
\end{aligned}
$$

The objective function can be minimized by using nonlinear optimization packages [20]. The optimization is the same as in the case of traditional $M$ channel cosine modulated filter banks [17].

Summarizing the results, we have shown that the system in Fig. 2 is a cosine modulated maximally decimated filter bank with approximate reconstruction property if the analysis and synthesis filters are chosen as in (12), $N=\left(2 m_{0}+1\right) M$ and the linear-phase prototype $P_{0}(z)$ is properly designed. 


\section{Linear Phase Cosine Modulated Filter BANKS WITH PERFECT RECONSTRUCTION}

Cosine modulated PR filter banks were reported in [11]-[13]. In [11], perfect reconstruction property is achieved by imposing some conditions on the polyphase components of the prototype filter so that the resulting filter bank is paraunitary. We will do something similar on the $2 M$ channel system. We will show that the filter bank in Fig. 2 with analysis and synthesis filters as in (12) is paraunitary and hence PR if the polyphase components of $P_{0}(z)$ satisfy some conditions to be derived in this section.

Let

$$
P_{0}(z)=\sum_{n=0}^{2 M-1} G_{n}\left(z^{2 M}\right) z^{-n}
$$

where $G_{n}(z)$ is the $n$th type 1 polyphase component of $P_{0}(z)$. Then

$$
U_{k}(z)=\sum_{n=0}^{2 M-1} G_{n}\left(z^{2 M}\right) z^{-n} W^{-k n} .
$$

Rewriting analysis filters in (3) in terms of polyphase components of $P_{0}(z)$ with $a_{k}, a_{k}^{\prime}$ and $N$ as determined in Section II, we obtain

$$
\begin{gathered}
H_{k}(z)=2 \sum_{n=0}^{2 M-1} G_{n}\left(z^{2 M}\right) z^{-n} \cos \left(\frac{\pi}{M} k n\right), \\
k=0,1, \ldots, M, \\
H_{k}^{\prime}(z)=2 z^{-M} \sum_{n=0}^{2 M-1} G_{n}\left(z^{2 M}\right) z^{-n} \sin \left(\frac{\pi}{M} k n\right), \\
k=1,2, \ldots, M-1 .
\end{gathered}
$$

Define the following $2 M$-component vectors

$$
\mathbf{h}(z)=\left(\begin{array}{c}
H_{0}(z) \\
\vdots \\
H_{M}(z) \\
H_{1}^{\prime}(z) \\
\vdots \\
H_{M-1}^{\prime}(z)
\end{array}\right), \quad \text { and } \quad \mathbf{f}(z)=\left(\begin{array}{c}
F_{0}(z) \\
\vdots \\
F_{M}(z) \\
F_{1}^{\prime}(z) \\
\vdots \\
F_{M-1}^{\prime}(z)
\end{array}\right)
$$

Using (14) the vector $h(z)$ can be written as

$$
\begin{array}{r}
\mathbf{h}(z)=\left(\begin{array}{cc}
\mathbf{I}_{M+1} & \mathbf{0} \\
\mathbf{0} & z^{-M} \mathbf{I}_{M-1}
\end{array}\right)\left(\begin{array}{cc}
\mathbf{C} & \Lambda_{1} \mathbf{C} \\
\mathbf{S} & \Lambda_{2} \mathbf{S}
\end{array}\right) \\
\left(\begin{array}{cc}
\mathbf{g}_{0}\left(z^{2 M}\right) & \mathbf{0} \\
\mathbf{0} & \mathbf{g}_{1}\left(z^{2 M}\right)
\end{array}\right)\left(\begin{array}{c}
\mathbf{e}(z) \\
z^{-M} \mathbf{e}(z)
\end{array}\right)
\end{array}
$$

where $\mathrm{g}_{i}(z)$ and $\Lambda_{i}$ are diagonal matrices with

$$
\left[\mathrm{g}_{0}(z)\right]_{k k}=G_{k}(z) \text {, }
$$

$$
\begin{aligned}
{\left[\mathbf{g}_{1}(z)\right]_{k k} } & =G_{k+M}(z), \quad k=0,1, \ldots, M-1 \\
{\left[\Lambda_{1}\right]_{k k} } & =(-1)^{k}, \quad k=0,1, \ldots, M \\
{\left[\Lambda_{2}\right]_{k k} } & =(-1)^{k}, \quad k=1,2, \ldots, M-1
\end{aligned}
$$

In addition, $\mathbf{C}$ and $\mathbf{S}$ are $(M+1) \times M$ and $(M-1) \times M$ matrices with

$$
\begin{aligned}
{[\mathbf{C}]_{m n}=} & \kappa_{m} \cos \left(\frac{\pi}{M} m n\right), \\
& m=0, \ldots, M, \quad n=0, \ldots, M-1
\end{aligned}
$$

and

$$
\begin{aligned}
{[\mathbf{S}]_{m n}=} & \sin \left(\frac{\pi}{M} m n\right), \\
& m=1, \ldots, M-1, \quad n=0, \ldots, M-1
\end{aligned}
$$

where

$$
\kappa_{m}= \begin{cases}\frac{1}{\sqrt{2}}, & \text { if } m=0 \text { or } M \\ 1, & \text { otherwise. }\end{cases}
$$

We can rearrange (15) and obtain

$$
\mathbf{h}(z)=\left(\begin{array}{cc}
\mathrm{Cg}_{0}\left(z^{2 M}\right) & \Lambda_{1} \mathbf{C g}_{1}\left(z^{2 M}\right) \\
z^{-2 M} \Lambda_{2} \mathbf{S g}_{1}\left(z^{2 M}\right) & \mathbf{S g}_{0}\left(z^{2 M}\right)
\end{array}\right)\left(\begin{array}{c}
\mathbf{e}(z) \\
z^{-M} \mathbf{e}(z)
\end{array}\right)
$$

The analysis bank has type I polyphase matrix [17] given by

$$
\mathbf{E}(z)=\left(\begin{array}{cc}
\mathrm{Cg}_{0}(z) & \Lambda_{1} \mathrm{Cg}_{1}(z) \\
z^{-1} \Lambda_{2} \operatorname{Sg}_{1}(z) & \operatorname{Sg}_{0}(z)
\end{array}\right)
$$

Since the synthesis filters are time-reversed versions of corresponding analysis filters (3), we can write $\mathrm{f}^{T}(z)=$ $z^{-(N+M)} \tilde{\mathbf{h}}(z)$. The synthesis bank has type II polyphase matrix given by

$$
\mathbf{R}(z)=z^{-(N+M)} \tilde{\mathbf{E}}(z)
$$

From [17], we know if $\mathbf{R}(z) \mathbf{E}(z)=c z^{-n_{0}} \mathbf{I}$ for some constant $c$ and nonnegative integer $n_{0}$, then the system in Fig. 2 is PR. By (20) and (21), we have (22), which appears at the bottom of this page. By exploiting the properties of $\mathbf{C}$ and $\mathbf{S}$ and using the same choice of $N$ as in (11), we obtain (23), which appears at the bottom of the next page) (see Appendix B for proof). In (23), $\mathrm{g}_{i}(z)$ is abbreviated $\mathrm{g}_{i}$ for convenience. The right-hand side of (23) is equal to $2 \mathbf{I}_{2 M}$ if and only if the following two conditions are true:

Condition $1: \quad \tilde{G}_{0}(z) G_{0}(z)=1$,

and $\quad \tilde{G}_{M}(z) G_{M}(z)=1$

Condition 2: $\quad \tilde{G}_{k}(z) G_{k}(z)+\tilde{G}_{k+M}(z) G_{k+M}(z)=2$,

for $\quad k=1,2, \ldots, M-1$.

Summarizing, we have the following theorem.

$$
\begin{aligned}
& z^{N+M} \mathbf{R}(z) \mathbf{E}(z)= \\
& \left(\begin{array}{cl}
\tilde{\mathbf{g}}_{0}(z) \mathbf{C}^{T} \mathbf{C} \mathbf{g}_{0}(z)+\tilde{\mathbf{g}}_{1}(z) \mathbf{S}^{T} \mathbf{S g}_{1}(z) & \tilde{\mathbf{g}}_{0}(z) \mathbf{C}^{T} \Lambda_{1} \mathbf{C g}_{1}(z)+z \tilde{\mathbf{g}}_{1}(z) \mathbf{S}^{T} \Lambda_{2} \mathbf{S g}_{0}(z) \\
\tilde{\mathbf{g}}_{1}(z) \mathbf{C}^{T} \Lambda_{1} \mathbf{C g}_{0}(z)+z^{-1} \tilde{\mathbf{g}}_{0}(z) \mathbf{S}^{T} \Lambda_{2} \mathbf{S g}_{1}(z) & \tilde{\mathbf{g}}_{1}(z) \mathbf{C}^{T} \mathbf{C g}_{1}(z)+\tilde{\mathbf{g}}_{0}(z) \mathbf{S}^{T} \mathbf{S} \mathbf{g}_{0}(z)
\end{array}\right)
\end{aligned}
$$


Theorem 3.1: If the above two conditions are imposed on $G_{k}(z)$ and, furthermore, the analysis and synthesis filters are as in (12) with the order of the prototype filter chosen as in (11), then the $2 M$ channel maximally decimated system in Fig. 2 has the following properties:

1) It is a cosine modulated system.

2) It has perfect reconstruction, i.e., $\hat{x}(n)=c x\left(n-n_{0}\right)$.

3) Each of the $2 M$ analysis and synthesis filters has linear phase.

4) The prototype filter $P_{0}(z)$ is Nyquist $(2 M)$ and $P_{0}(z) \tilde{P}_{0}(z)$ is also Nyquist $(2 M)$.

Remark on Theorem 3.1: In the thoerem, we have constrained the order of the prototype $N$ to be an odd multiple of $M$. It can be shown that the theorem can be extended to the case when $N+M$ is even.

1) Nonzero Samples and Free Parameters of $P_{0}(z)$ : Although $P_{0}(z)$ is of order $N$, the number of free parameters in $P_{0}(z)$ is only about $N / 4$ for reasons to be explained below. Notice that with $N=\left(2 m_{0}+1\right) M$ the first $M+1$ polyphase components of $P_{0}(z)$ have order $m_{0}$ and the last $M-1$ polyphase components of $P_{0}(z)$ have order $m_{0}-1$. Suppose that $G_{k}(z)$ has impulse reponse $g_{k}(n)$. To satisfy (25), it can be verified that $g_{k}(0)$ or $g_{k}\left(m_{0}\right)$ must be zero for $k=1,2, \ldots, M-1$. From (24) we see that $G_{0}(z)$ and $G_{M}(z)$ are merely delays, i.e., of the form $c z^{-n_{0}}$. The nonzero samples and free parameters of $P_{0}(z)$ are discussed in two cases: odd $M$ and even $M$.

Case $1 M$ is Odd: Both $G_{k}(z)$ and $G_{k+M}(z)$, for $k=$ $1,2, \ldots, M-1$ have $m_{0}$ nonzero samples. As a result, $P_{0}(z)$ has $2 m_{0}(M-1)+2$ nonzero samples. Since $P_{0}(z)$ is linearphase, the polyphase components $G_{k}(z)$ and $G_{M-k}$, for $k=$ $1,2, \ldots, M-1$ are time-reversed versions of each other. So are $G_{k+M}(z)$ and $G_{2 M-k}$ for $k=1,2, \ldots, M-1$. These constraints reduce the number of free parameters to about $N / 2$. But $G_{k+M}(z)$ is also related to $G_{k}(z)$ by $(25)$. The number of free parameters is again cut down by half. The number of free parameters for $P_{0}(z)$ is only $(M-1) m_{0} / 2 \approx N / 4$.

Case $2 M$ is Even: In this case, $G_{k}(z)$ are constrained as in odd $M$ case with $G_{\frac{M}{2}}(z)$ and $G_{\frac{3 M}{2}}(z)$ satisfying additional conditions. By (25), ${ }^{2} G_{\frac{M}{2}}(z)$ and ${ }^{2} G_{\frac{3 M}{2}}(z)$ form a power complementary pair and they both have linear phase because $P_{0}(z)$ is linear-phase. As a result, $G_{\frac{M}{2}}(z)$ and $G_{\frac{3 M}{2}}(z)$ are further constrained in the following:

$$
\left\{\begin{array}{lll}
\text { even } m_{0}, \tilde{G}_{\frac{M}{2}}(z) G_{\frac{M}{2}}(z)=2, & \text { and } & G_{\frac{3 M}{2}}(z)=0, \\
\text { odd } m_{0}, G_{\frac{M}{2}}(z)=0 & \text { and } \tilde{G}_{\frac{3 M}{2}}(z) G_{\frac{3 M}{2}}(z)=2 .
\end{array}\right.
$$

In the even $M$ case, $P_{0}(z)$ has $2 m_{0}(M-2)+1$ nonzero samples and free parameters $\frac{M-2}{2} m_{0}$, which is equal to the

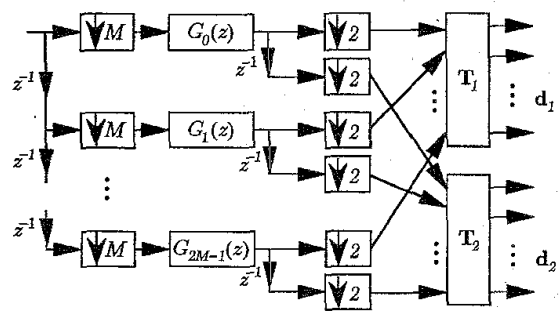

Fig. 5. Implementation of the $2 M$ channel system. Both $T_{1}$ and $T_{2}$ in the figure are of dimension $M$ by $2 M$.

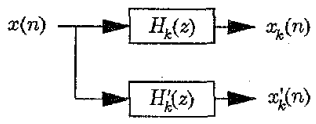

(a)

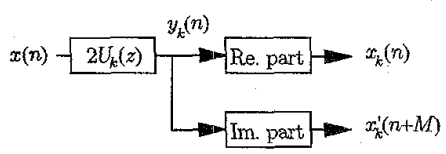

(b)

Fig. 6. Interpretation of $x_{k}(n)$ and $x_{k}^{\prime}(n+M), k=1,2, \ldots, M-1$.

total number of parameters for $M-1$ case and is also close to $N / 4$. Therefore, when we increase the number of channels from $2(M-1)$ to $2 M$ (where $M-1$ is odd), the number of free parameters for $P_{0}(z)$ does not increase.

2) Design Complexity: Since the system in this case is PR, it is sufficient to minimize the stopband energy of the prototype filter under the two conditions in (24) and (25). This is similar to the case of traditional $M$ channel cosine modulated filter banks [17].

\section{EFFICIENT IMPLEMENTATION OF THE LINEAR Phase COSINE MOdulated FILTER BANK}

The implementation cost of a conventional $M$ channel maximally decimated cosine modulated filter bank is that of the prototype filter plus one DCT matrix working at $M$-fold decimated rate [17]. We will show that the proposed $2 M$ channel linear phase cosine modulated system in Fig. 2 has nearly the same cost, i.e., number of computations per input sample is nearly the same. The implementation proposed here can be applied to both the approximate PR case (Section II) and the PR case (Section III).

Define two $M \times 2 M$ matrices

$$
\mathbf{T}_{1}=\left(\begin{array}{ll}
\mathrm{C} & \Lambda_{1} \mathbf{C}
\end{array}\right) \quad \text { and } \quad \mathbf{T}_{2}=\left(\begin{array}{ll}
\mathbf{S} & \Lambda_{2} \mathbf{S}
\end{array}\right)
$$

$$
\begin{aligned}
& \frac{2 z^{N+M}}{M} \mathbf{R}(z) \mathbf{E}(z)= \\
& \left(\begin{array}{cc}
\tilde{\mathbf{g}}_{0}\left(\begin{array}{cc}
2 & 0 \\
0 & \mathbf{I}_{M-1}
\end{array}\right) \mathbf{g}_{0}+\tilde{\mathrm{g}}_{1}\left(\begin{array}{cc}
0 & 0 \\
0 & \mathbf{I}_{M-1}
\end{array}\right) \mathbf{g}_{1} & \\
0 & \tilde{\mathrm{g}}_{1}\left(\begin{array}{cc}
2 & 0 \\
0 & \mathbf{I}_{M-1}
\end{array}\right) \mathbf{g}_{1}+\tilde{\mathrm{g}}_{0}\left(\begin{array}{cc}
0 & 0 \\
0 & \mathbf{I}_{M-1}
\end{array}\right) \cdot \mathbf{g}_{0}
\end{array}\right)
\end{aligned}
$$




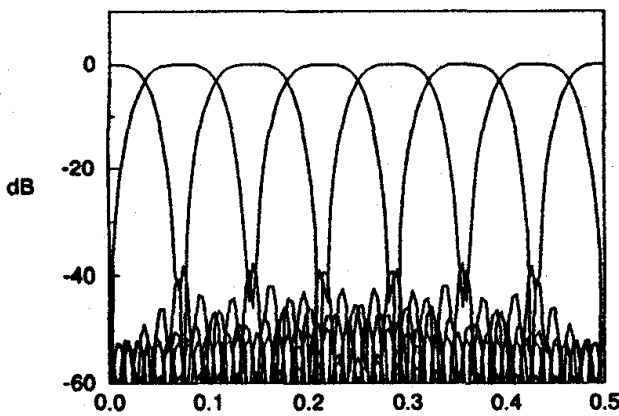

(a)

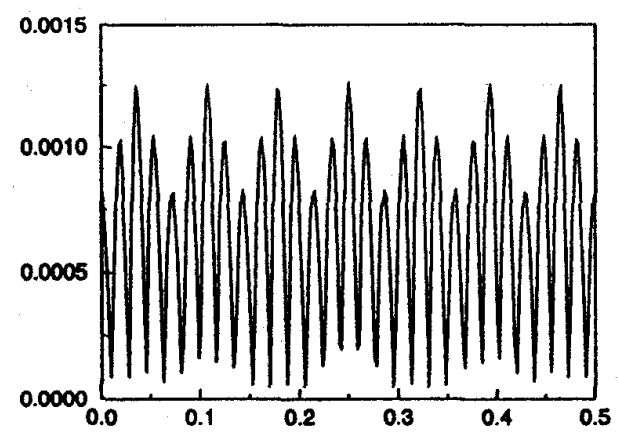

(c)

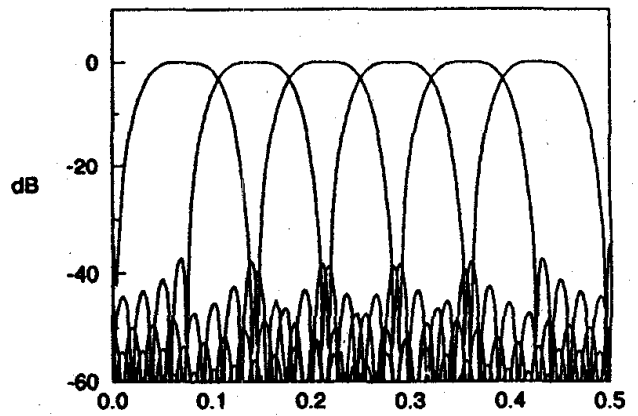

(b)

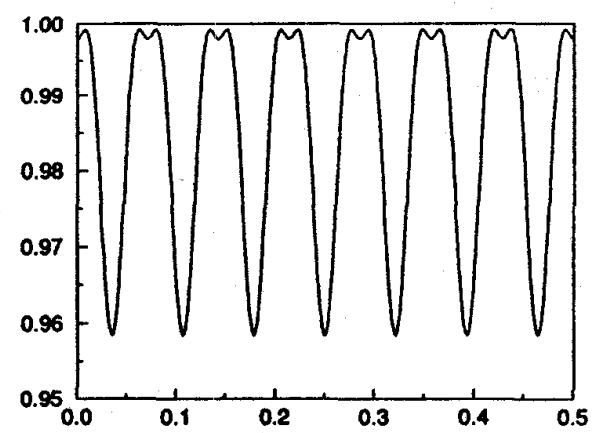

(d)

Fig. 7. Example 6.1-Approximate PR system: (a) Normalized magnitude responses of the first set of analysis filters; (b) magnitude responses of the second set of analysis filters; (c) plot of aliasing error $\sqrt{\sum_{i=1}^{i=2 M-1}\left|A_{i}\left(e^{j \omega}\right)\right|^{2}}$; (d) plot of amplitude distortion function $M\left|T\left(e^{j \omega}\right)\right|$.

where $\mathbf{C}, \mathbf{S}, \Lambda_{1}$, and $\Lambda_{2}$ are as defined in (17) and (18). From (15), we can draw Fig. 5, the implementation of the $2 M$ channel cosine modulated system. The input to $\mathbf{T}_{1}, \mathbf{a}(n)$ is partitioned according to $\mathbf{a}(n)=\left(\begin{array}{l}\mathbf{a}_{1}(n) \\ \mathbf{a}_{2}(n)\end{array}\right)$. Similarly, $\mathbf{b}(n)$, which is the input vector to $\mathbf{T}_{2}$, is partitioned as $\left(\begin{array}{l}\mathbf{b}_{1}(n) \\ \mathbf{b}_{2}(n)\end{array}\right)$. The vectors $\mathbf{a}_{1}(n), \mathbf{a}_{2}(n), \mathbf{b}_{1}(n)$, and $\mathbf{b}_{2}(n)$ are all of dimension $M \times 1$. Their dependence on $n$ will be dropped for convenience. As indicated in Fig. 5 outputs of $\mathbf{T}_{1}$ and $\mathbf{T}_{2}$ are $d_{1}$ and $d_{2}$, respectively.

$$
\mathbf{d}_{1}=\mathbf{T}_{1} \mathbf{a}, \quad \text { and } \quad \mathbf{d}_{2}=\mathbf{T}_{2} \mathbf{b} .
$$

They can also be expressed in terms of $\mathbf{a}_{1}, \mathbf{a}_{2}, \mathbf{b}_{1}$, and $\mathbf{b}_{2}$ as (Appendix C)

$$
\begin{aligned}
& \mathbf{d}_{1}=\mathbf{C}\left(\mathbf{a}_{1}+\left(\begin{array}{cc}
0 & \mathbf{0} \\
\mathbf{0} & \mathbf{J}_{M-1}
\end{array}\right) \mathbf{a}_{2}\right)+\sqrt{2}\left[\mathbf{a}_{2}\right]_{0} \mathbf{r} \\
& \mathbf{d}_{1}=\mathbf{S}\left(\mathbf{b}_{1}-\left(\begin{array}{cc}
0 & \mathbf{0} \\
\mathbf{0} & \mathbf{J}_{M-1}
\end{array}\right) \mathbf{b}_{2}\right)
\end{aligned}
$$

where $\left[\mathbf{a}_{2}\right]_{0}$ is the 0 th element of $\mathbf{a}_{2}$, and $\mathbf{r}$ is $\sqrt{\frac{M}{2}}$ the last column of $\mathbf{C}$. From (26), we observe that the major computation in $\mathbf{T}_{1}$ is only the matrix $\mathbf{C}$ and the major computation in $\mathbf{T}_{2}$ is the matrix $\mathbf{S}$. Matrices $\mathbf{C}$ and $\mathbf{S}$ can be implemented by fast algorithms for DCT and DST matrices [21]. But both computations are done after $2 M$-fold decimation, which is equivalent to computing one matrix after $M$-fold decimation. That is the same as the case in a conventional $M$ channel maximally decimated cosine modulated filter bank [17]. The implementation of synthesis bank is similar.

Notice that quantization of filter coefficients will not affect the relation that $G_{k}(z)$ is the time-reversed version of $G_{M-k}(z)$, for $k=1,2, \ldots, M-1$. Similarly for $G_{2 M-k}(z)$ and $G_{k+M}(z)$, for $k=1,2, \ldots, M-1$. We conclude that the linear phase property of the individual analysis and synthesis filters is preserved in spite of filter coefficient quantization.

\section{Subband Signals, Coding Gain, AND OPTIMAL BIT ALLOCATION}

In a traditional $N$-channel subband coding system, the output $x_{k}(n)$ of the $k$ th analysis filter has total bandwidth $2 \pi / N$ in $[-\pi, \pi)$ and is decimated by $N$. This decimation does not cause aliasing except for the reason that the analysis filters are not ideal bandlimiting functions and cannot have infinitely sharp roll-off. This aliasing created due to practical limitations of filters is cancelled by the choice of synthesis filters.

In the 2M-channel maximally decimated system of Fig. 2, however, the situation is very unusual. Each analysis filter has a total bandwidth of $2 \pi / M$ in $[-\pi, \pi)$ [except $H_{0}(z)$ and $\left.H_{M}(z)\right]$ and yet its output is decimated by $2 M$. This means there will be severe aliasing even if the filters were ideal bandpass filters. Even this aliasing is cancelled by appopriate choice of synthesis filters, as already proved in the preceding sections.

In the context of subband quantization and coding, one wonders how this system would perform: with such severe aliasing in the subbands, would it still be possible to obtain 


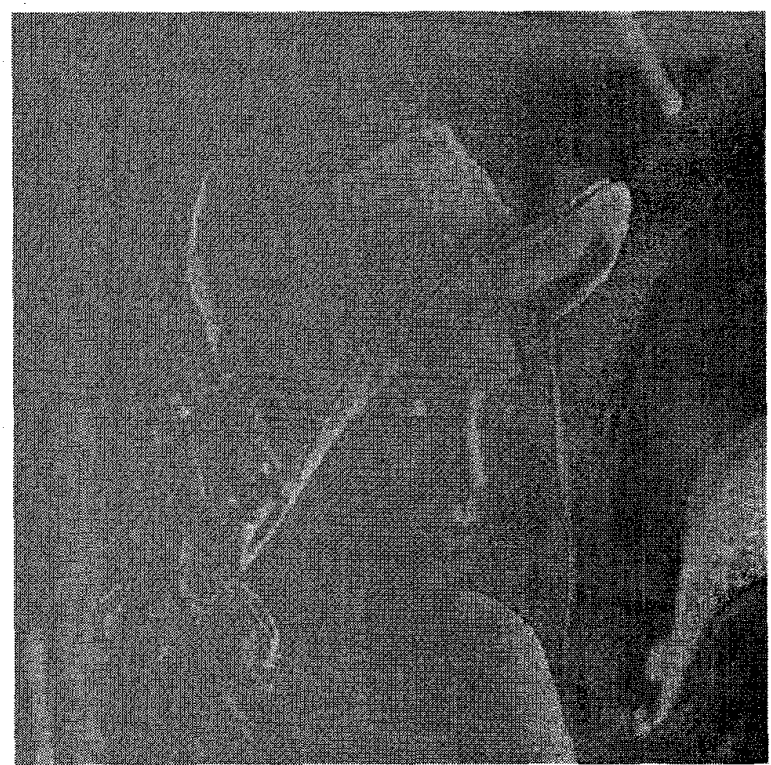

(a)

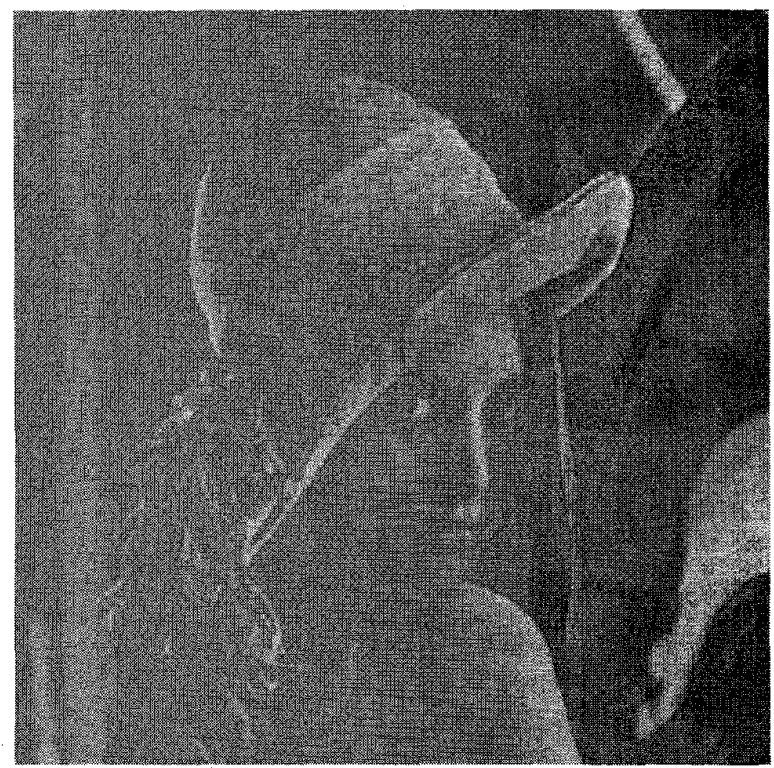

(b)

Fig. 8. Example 6.2. Image coding example with the linear phase subband coder design in Example 6.1: (a) Image of "Lenna" with 8 b/pixel; (b) reconstructed version from subbands. The PSNR is $35.5 \mathrm{~dB}$, and the subband bit rate is $0.35 \mathrm{~b}$ /pixel (bit allocation followed by entropy coding).

the usual coding gain advantage? That is, would it still be possible to exploit the energy distribution of the original input signal $x(n)$ in the usual way? In short, does the proposed filter bank scheme make sense as a subband coder? We now look deeper into this important aspect.

\section{A. An Interpretation of the Subband Signals}

To explain this, consider the filter bank in Fig. 2. Suppose $x(n)$ is the input. Let $x_{k}(n)$ and $x_{k}^{\prime}(n)$ denote the outputs of

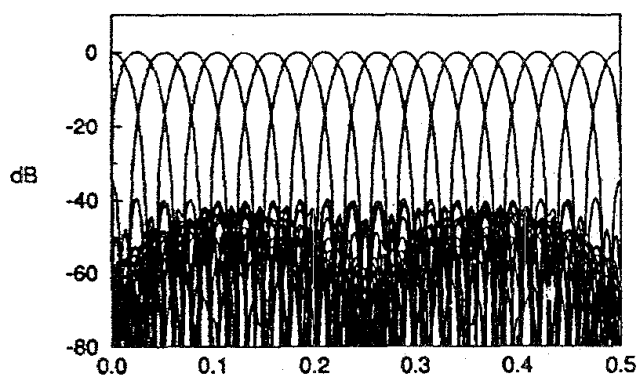

(a)

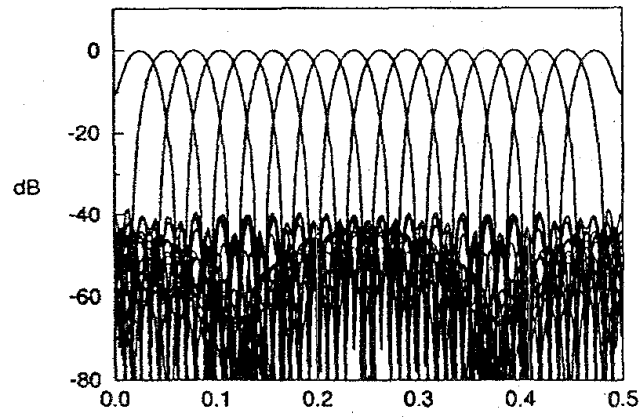

(b)

Fig. 9. Example 6.3. Cosine modulated PR system: (a) Normalized magnitude responses of the first set of analysis filters; (b) magnitude responses of the second set of analysis filters.

$H_{k}(z)$ and $H_{k}^{\prime}(z)$ respectively, as in Fig. 6(a). With the filters constructed as in (12), we obtain

$$
\begin{aligned}
& X_{k}(z)=X(z)\left(U_{k}(z)+U_{-k}(z)\right), \quad k=0,1, \ldots, M \\
& X_{k}^{\prime}(z)=z^{-M} X(z)\left(-j U_{k}(z)+j U_{-k}(z)\right), \\
& \quad k=1,2, \ldots, M_{-1}
\end{aligned}
$$

where $j=\sqrt{-1}$. With (27) and (28), we get the following expression:

$$
\begin{gathered}
2 z^{-M} X(z) U_{k}(z)=z^{-M} X_{k}(z)+j X_{k}^{\prime}(z) \\
k=1, \ldots, M-1
\end{gathered}
$$

If we take a real signal $x(n)$ as the input of the complex coefficient filter $2 z^{-M} U_{k}(z)$, then the output is the right hand side of (29). The output represents the energy of $x(n)$ in the $U_{k}(z)$ subband (Fig. 4). From (29), we observe that the output of $2 U_{k}(z)$ has real part $x_{k}(n)$ and imaginary part $x_{k}^{\prime}(n+M)$. Fig. 6(b) illustrates this relation.

Thus, except for delays and scale factors, the signals $x_{k}(n)$ and $x_{k}^{\prime}(n)$ can be interpreted as the real and imaginary parts of the one-sided (hypothetical) complex subband signal $y_{k}(n)$ (which is analogous to the analytic signal of $x_{k}(n)$ [22]). Therefore, $x_{k}(n)$ and $x_{k}^{\prime}(n+M)$ together retain the usual meaning of subband signals. From the fact that $H_{k}(z)$ and $H_{k}^{\prime}(z)$ have the same spectral occupancy (Fig. 3), we see that the energies of $x_{k}(n)$ and $x_{k}^{\prime}(n)$ are essentially the same. These, in turn, are proportional to the energy of the hypothetical complex subband signal $y_{k}(n)$. The decimation of 
TABLE I

Prototype Filters with Stopband Attenuation about 25 dB and Order $3 M$
n
$\mathrm{M}=7$
$M=8$
$\mathbf{M}=9$
$\mathbf{M}=11$
$\mathbf{M}=13$
$\mathrm{M}=15$

\begin{tabular}{|c|c|c|c|c|c|c|}
\hline 0 & $0.0000000 \mathrm{E}+00$ & $0.0000000 \mathrm{E}+00$ & $0.0000000 \mathrm{E}+00$ & $0.0000000 \mathrm{E}+00$ & $0.0000000 \mathrm{E}+00$ & $0.0000000 \mathrm{E}+00$ \\
\hline 1 . & $0.0000000 \mathrm{E}+00$ & $0.0000000 \mathrm{E}+00$ & $0.0000000 \mathrm{E}+00$ & $0.0000000 \mathrm{E}+00$ & $0.0000000 \mathrm{E}+00$ & $0.0000000 \mathrm{E}+00$ \\
\hline 2 & $0.0000000 \mathrm{E}+00$ & $0.0000000 \mathrm{E}+00$ & $0.0000000 \mathrm{E}+00$ & $0.0000000 \mathrm{E}+00$ & $0.0000000 \mathrm{E}+00$ & $0.0000000 \mathrm{E}+00$ \\
\hline 3 & $0.0000000 \mathrm{E}+00$ & $0.0000000 \mathrm{E}+00$ & $0.0000000 \mathrm{E}+00$ & $0.0000000 \mathrm{E}+00$ & $0.0000000 \mathrm{E}+00$ & $0.0000000 \mathrm{E}+00$ \\
\hline 4 & $1.8732471 \mathrm{E}-02$ & $0.0000000 \mathrm{E}+00$ & $0.0000000 \mathrm{E}+00$ & $0.0000000 \mathrm{E}+00$ & $0.0000000 \mathrm{E}+00$ & $0.0000000 \mathrm{E}+00$ \\
\hline 5 & $3.5665461 \mathrm{E}-02$ & $1.8383085 \mathrm{E}-02$ & $1.3193124 \mathrm{E}-02$ & $0.0000000 \mathrm{E}+00$ & $0.0000000 \mathrm{E}+00$ & $0.0000000 \mathrm{E}+00$ \\
\hline 6 & $5.6870395 \mathrm{E}-02$ & $3.2890536 \mathrm{E}-02$ & $2.2634022 \mathrm{E}-02$ & $1.0140769 \mathrm{E}-02$ & $0.0000000 \mathrm{E}+00$ & $0.0000000 \mathrm{E}+00$ \\
\hline 7 & $7.8559943 \mathrm{E}-02$ & $5.1283753 \mathrm{E}-02$ & $3.4499846 \mathrm{E}-02$ & $1.6130896 \mathrm{E}-02$ & $8.2246300 \mathrm{E}-03$ & $0.0000000 \mathrm{E}+00$ \\
\hline 8 & $9.5441539 \mathrm{E}-02$ & $7.0416880 \mathrm{E}-02$ & $4.7970428 \mathrm{E}-02$ & $2.3511272 \mathrm{E}-02$ & $1.2364566 \mathrm{E}-02$ & $6.8684742 \mathrm{E}-03$ \\
\hline 9 & $1.0522026 \mathrm{E}-01$ & $8.5364224 E-02$ & $6.1201399 \mathrm{E}-02$ & $3.2084683 \mathrm{E}-02$ & $1.7353344 \mathrm{E}-02$ & $9.8544667 \mathrm{E}-03$ \\
\hline 10 & $1.0950993 \mathrm{E}-01$ & $9.3996206 \mathrm{E}-02$ & $7.2042075 \mathrm{E}-02$ & $4.1267157 \mathrm{E}-02$ & $2.3139003 \mathrm{E}-02$ & $1.3401593 \mathrm{E}-02$ \\
\hline 11 & & $9.7873062 \mathrm{E}-02$ & $7.9378732 \mathrm{E}-02$ & $5.0105611 \mathrm{E}-02$ & $2.9520914 \mathrm{E}-02$ & $1.7506612 \mathrm{E}-02$ \\
\hline 12 & & $9.9584507 \mathrm{E}-02$ & $8.3539952 \mathrm{E}-02$ & $5.7603527 \mathrm{E}-02$ & $3.6116001 \mathrm{E}-02$ & $2.2101899 \mathrm{E}-02$ \\
\hline 13 & & & $8.5540423 \mathrm{E}-02$ & $6.3180042 \mathrm{E}-02$ & $4.2403735 \mathrm{E}-02$ & $2.7027928 \mathrm{E}-02$ \\
\hline 14 & & & & $6.6845827 \mathrm{E}-02$ & $4.7872622 \mathrm{E}-02$ & $3.2028343 \mathrm{E}-02$ \\
\hline 15 & & & & $6.8999556 \mathrm{E}-02$ & $5.2198363 \mathrm{E}-02$ & $3.6784105 \mathrm{E}-02$ \\
\hline 16 & & . & & $7.0130659 \mathrm{E}-02$ & $5.5323955 \mathrm{E}-02$ & $4.0991781 \mathrm{E}-02$ \\
\hline 17 & & & & & $5.7402221 \mathrm{E}-02$ & $4.4448081 \mathrm{E}-02$ \\
\hline 18 & & & & & $5.8679392 \mathrm{E}-02$ & $4.7091898 \mathrm{E}-02$ \\
\hline 19 & & & & & $5.9401254 \mathrm{E}-02$ & $4.8986318 \mathrm{E}-02$ \\
\hline 20 & & & & & & $5.0264681 \mathrm{E}-02$ \\
\hline 21 & & & & & & $5.1078668 \mathrm{E}-02$ \\
\hline 22 & & & & & & $5.1565152 \mathrm{E}-02$ \\
\hline$n$ & $M=16$ & $M=17$ & $\mathbf{M}=$ & & $M=21$ & $M=24$ \\
\hline
\end{tabular}

\begin{tabular}{|c|c|c|c|c|c|}
\hline 0 & $0.0000000 E+00$ & $0,0000000 E+00$ & $0.0000000 \mathrm{E}+00$ & $0.0000000 \mathrm{E}+00$ & $0.0000000 \mathrm{E}+00$ \\
\hline 1 & $0.0000000 \mathrm{E}+00$ & $0.0000000 \mathrm{E}+00$ & $0.0000000 \mathrm{E}+00$ & $0.0000000 \mathrm{E}+00$ & $0.0000000 \mathrm{E}+00$ \\
\hline 2 & $0.0000000 \mathrm{E}+00$ & $0.0000000 \mathrm{E}+00$ & $0.0000000 \mathrm{E}+00$ & $0.0000000 \mathrm{E}+00$ & $0.0000000 \mathrm{E}+00$ \\
\hline 3 & $0.0000000 \mathrm{E}+00$ & $0.0000000 \mathrm{E}+00$ & $0.0000000 \mathrm{E}+00$ & $0.0000000 \mathrm{E}+0.0$ & $0.0000000 \mathrm{E}+00$ \\
\hline 4 & $0.0000000 \mathrm{E}+00$ & $0.0000000 \mathrm{E}+00$ & $0.0000000 \mathrm{E}+00$ & $0.0000000 \mathrm{E}+00$ & $0.0000000 \mathrm{E}+00$ \\
\hline 5 & $0.0000000 \mathrm{E}+00$ & $0.0000000 \mathrm{E}+00$ & $0.0000000 \mathrm{E}+00$ & $0.0000000 \mathrm{E}+00$ & $0.0000000 \mathrm{E}+00$ \\
\hline 6 & $0.0000000 \mathrm{E}+00$ & $0.0000000 \mathrm{E}+00$ & $0.0000000 \mathrm{E}+00$ & $0.0000000 \mathrm{E}+00$ & $0.0000000 \mathrm{E}+00$ \\
\hline 7 & $0.0000000 \mathrm{E}+00$ & $0.0000000 E+00$ & $0.0000000 \mathrm{E}+00$ & $0.0000000 \mathrm{E}+00$ & $0.0000000 \mathrm{E}+00$ \\
\hline 8 & $0.0000000 \mathrm{E}+00$ & $0.0000000 \mathrm{E}+00$ & $0.0000000 \mathrm{E}+00$ & $0.0000000 \mathrm{E}+00$ & $0.0000000 \mathrm{E}+00$ \\
\hline 9 & $-7.0567914 \mathrm{E}-03$ & $5.9379618 \mathrm{E}-03$ & $0.0000000 \mathrm{E}+00$ & $0.0000000 \mathrm{E}+00$ & $0.0000000 \mathrm{E}+00$ \\
\hline 10 & $-9.9461336 \mathrm{E}-03$ & $8.2342790 \mathrm{E}-03$ & $5.1904905 \mathrm{E}-03$ & $0.0000000 \mathrm{E}+00$ & $0.0000000 \mathrm{E}+00$ \\
\hline 11 & $-1.3300380 \mathrm{E}-02$ & $1.0910574 \mathrm{E}-02$ & $6.9789570 \mathrm{E}-03$ & $4.6321577 \mathrm{E}-03$ & $0.0000000 \mathrm{E}+00$ \\
\hline 12 & $-1.7161896 \mathrm{E}-02$ & $1.3968583 \mathrm{E}-02$ & $9.0403012 \mathrm{E}-03$ & $6.0823399 \mathrm{E}-03$ & $0.0000000 \mathrm{E}+00$ \\
\hline 13 & $-2.1408026 \mathrm{E}-02$ & $1.7380301 \mathrm{E}-02$ & $1.1381438 \mathrm{E}-02$ & $7.7328479 \mathrm{E}-03$ & $4.0860508 \mathrm{E}-03$ \\
\hline 14 & $-2.5932379 \mathrm{E}-02$ & $2.1075551 \mathrm{E}-02$ & $1.3993922 \mathrm{E}-02$ & $9.5890298 \mathrm{E}-03$ & $5.3841990 \mathrm{E}-03$ \\
\hline 15 & $-3.0482434 \mathrm{E}-02$ & $2.4932616 \mathrm{E}-02$ & $1.6846952 \mathrm{E}-02$ & $1.1647991 \mathrm{E}-02$ & $6.8056680 \mathrm{E}-03$ \\
\hline 16 & $-3.4808738 \mathrm{E}-02$ & $2.8784655 \mathrm{E}-02$ & $1.9880926 \mathrm{E}-02$ & $1.3894873 \mathrm{E}-02$ & $8.3809124 \mathrm{E}-03$ \\
\hline 17 & $-3.8653819 \mathrm{E}-02$ & $3.2440869 \mathrm{E}-02$ & $2.3005024 \mathrm{E}-02$ & $1.6299068 \mathrm{E}-02$ & $1.0060428 \mathrm{E}-02$ \\
\hline 18 & $-4.1842661 \mathrm{E}-02$ & $3.5724832 \mathrm{E}-02$ & $2.6102687 \mathrm{E}-02$ & $1.8811663 \mathrm{E}-02$ & $1.1857825 \mathrm{E}-02$ \\
\hline 19 & $-4.4328241 \mathrm{E}-02$ & $3.8512137 \mathrm{E}-02$ & $2.9045232 \mathrm{E}-02$ & $2.1365729 \mathrm{E}-02$ & $1.3719700 \mathrm{E}-02$ \\
\hline 20 & $-4.6138550 \mathrm{E}-02$ & $4.0750965 \mathrm{E}-02$ & $3.1715938 \mathrm{E}-02$ & $2.3880869 \mathrm{E}-02$ & $1.5641153 \mathrm{E}-02$ \\
\hline 21 & $-4.7396165 \mathrm{E}-02$ & $4.2458746 \mathrm{E}-02$ & $3.4029691 \mathrm{E}-02$ & $2.6272669 \mathrm{E}-02$ & $1.7568068 \mathrm{E}-02$ \\
\hline 22 & $-4.8211730 \mathrm{E}-02$ & $4.3700099 \mathrm{E}-02$ & $3.5944399 \mathrm{E}-02$ & $2.8464194 \mathrm{E}-02$ & $1.9479842 \mathrm{E}-02$ \\
\hline 23 & $-4.8718561 \mathrm{E}-02$ & $4.4562085 \mathrm{E}-02$ & $3.7462398 \mathrm{E}-02$ & $3.0397564 \mathrm{E}-02$ & $2.1328626 \mathrm{E}-02$ \\
\hline 24 & $-4.9226990 \mathrm{E}-02$ & $4.5133321 \mathrm{E}-02$ & $3.8618922 \mathrm{E}-02$ & $3.2041030 \mathrm{E}-02$ & $2.3085849 \mathrm{E}-02$ \\
\hline 25 & & $4.5492424 \mathrm{E}-02$ & $3.9467884 \mathrm{E}-02$ & $3.3389319 \mathrm{E}-02$ & $2.4718467 \mathrm{E}-02$ \\
\hline 26 & & & $4.0068990 \mathrm{E}-02$ & $3.4459233 \mathrm{E}-02$ & $2.6200165 \bar{E}-02$ \\
\hline 27 & & & $4.0478947 \mathrm{E}-02$ & $3.5282156 \mathrm{E}-02$ & $2.7518646 \mathrm{E}-02$ \\
\hline 28 & & & $4.0746900 \mathrm{E}-02$ & $3.5896473 \mathrm{E}-02$ & $2.8657760 \mathrm{E}-02$ \\
\hline 29 & & & & $3.6341565 \mathrm{E}-02$ & $2.9625711 \mathrm{E}-02$ \\
\hline 30 & & & & $3.6653941 \mathrm{E}-02$ & $3.0418824 \mathrm{E}-02$ \\
\hline 31 & & & & $3.6865287 \mathrm{E}-02$ & $3.1059631 \mathrm{E}-02$ \\
\hline 32 & & & & & $3.1554290 \mathrm{E}-02$ \\
\hline 33 & & & & & $3.1931110 \mathrm{E}-02$ \\
\hline 34 & & & & & $3.2201293 \mathrm{E}-02$ \\
\hline 35 & & & & & $3.2391621 \mathrm{E}-02$ \\
\hline 36 & & & & & $3.2648321 \mathrm{E}-02$ \\
\hline
\end{tabular}

$x_{k}(n)$ and $x_{k}^{\prime}(n)$ by $2 M$ is equivalent to decimating $y_{k}(n)$ by $2 M$. Because $y_{k}(n)$ has bandwidth $2 \pi / 2 M$, the decimation of $y_{k}(n)$ by $2 M$ does not lead to severe aliasing other than due to usual filter nonidealities. Therefore, even though the decimation of the subband signals in Fig. 2 creates severe aliasing, it still makes sense to quantize and encode the decimated signals based on the energy distributions of the undecimated signals. In Section VI, we provide an image coding example (Example
6.2) that shows that subband quantization and reconstruction work in the usual way. We now proceed to give the quantitative details.

\section{B. Coding Gain}

In Section III, the type I polyphase matrix of the analysis bank is constrained to be paraunitary, i.e., $\tilde{\mathbf{E}}(z) \mathbf{E}(z)=$ $c z^{-n_{0}} \mathbf{I}$, so that the system in Fig. 2 is PR. The resulting new 
cosine modulated filter bank with perfect reconstruction falls into the category of paraunitary filter banks. The coding gain and optimal bit allocation for paraunitary systems can be found in [19].

Suppose that the real input signal $x(n)$ has power spectral density $S_{x x}(\omega)$ and variance $\sigma_{x}^{2}$. Let $\sigma_{x_{k}}^{2}$ be the variance of $x_{k}(n)$ and $\sigma_{x_{k}^{\prime}}^{2}$ be the variance of $x_{k}^{\prime}(n)$.

$$
\begin{aligned}
& \sigma_{x_{k}}^{2}=\frac{1}{2 \pi} \int_{0}^{2 \pi} S_{x x}(\omega)\left|H_{k}\left(e^{j \omega}\right)\right|^{2} d \omega \\
& \sigma_{x_{k}^{\prime}}^{2}=\frac{1}{2 \pi} \int_{0}^{2 \pi} S_{x x}(\omega)\left|H_{k}^{\prime}\left(e^{j \omega}\right)\right|^{2} d \omega .
\end{aligned}
$$

A $2 M$ channel paraunitary filter bank in Fig. 2 has coding gain $G_{2 M}$ [19]

$$
G_{2 M}=\frac{\sigma_{x}^{2}}{\left(\prod_{k=0}^{M} \sigma_{x_{k}}^{2} \prod_{k=1}^{M-1} \sigma_{x_{k}^{\prime}}^{2}\right)^{1 / 2 M}} .
$$

Assume that the prototype filter $P_{0}(z)$ has large enough stopband attenuation and $U_{k}(z)$ and $U_{-k}(z)$ do not overlap in passbands, i.e., $\left|U_{k}\left(e^{j \omega}\right) U_{-k}\left(e^{j \omega}\right)\right| \approx 0$ (see Fig. 4). From (30) and (31), we have

$$
\sigma_{x_{k}}^{2} \approx \sigma_{x_{k}^{\prime}}^{2}, \quad k=1,2, \ldots, M-1
$$

Equation (32) then becomes

$$
G_{2 M} \approx \frac{\sigma_{x}^{2}}{\left(\sigma_{x_{0}}^{2} \sigma_{x_{M}}^{2}\right)^{1 / 2 M}\left(\prod_{k=1}^{M-1} \sigma_{x_{k}}^{2}\right)^{1 / M}}
$$

which is closer to the coding gain of $M$ channel rather than $2 M$ channel paraunitary systems.

\section{Optimal Bit Allocation}

In a conventional $M$ channel filter bank with quantizers in the subbands, bits are allocated according to the energy of subband signals [17]. Let $\hat{x}_{k}(n)$ and $\hat{x}_{k}^{\prime}(n)$ be decimated signals of $x_{k}(n)$ and $x_{k}^{\prime}(n)$. Since decimators do not change signal variances, we have $\sigma_{\hat{x}_{k}}^{2}=\sigma_{x_{k}}^{2}$ and $\sigma_{\hat{x}_{k}^{\prime}}^{2}=\sigma_{x_{k}^{\prime}}^{2}$. Allocating bits according to $\sigma_{\hat{x}_{k}}^{2}$ and $\sigma_{\hat{x}_{k}^{\prime}}^{2}$ is equivalent to allocating bits according to $\sigma_{x_{k}}^{2}$ and $\sigma_{x_{k}^{\prime}}^{2}$, which represent the energy of the subband signals. Therefore, the idea of optimal bit allocation in this $2 M$ channel system is the same as the conventional case.

In the approximate PR case, if we ignore the residual aliasing and reconstruction errors, we can use the above formula for coding gain and optimal bit allocation.

\section{NUMERICAL EXAMPLES AND TABLES of PRototype FULTER COEFFICIENTS}

We now present two design examples. This will be followed by tables of prototype filter coefficients for the PR case.

All of them are obtained by using nonlinear optimization programs in [20].
Example 6.1: Approximately PR system with $M=7$, i.e., 14 channels. The prototype filter has order $N=49$, stopband attenuation $39 \mathrm{~dB}$, and stopband edge $\omega_{s}=0.133 \pi$. Fig. 7(a) and (b) show, respectively, magnitude responses of the first set of analysis filters and the second set of analysis filters. (In Fig. 7(a) magnitude responses are all normalized with maximum value of $0 \mathrm{~dB}$.) Fig. 7(c) is a plot of aliasing error $\sqrt{\sum_{i=1}^{i=2 M-1}\left|A_{i}\left(e^{j \omega}\right)\right|^{2}}$. Aliasing error is suppressed satisfactorily; worst peak aliasing error is very small, only about 0.0013. We can see from Fig. 7(d) that the amplitude distortion function is approximately flat with peak amplitude distortion 0.02 .

Example 6.2-Image Coding: For this, a 2-D separable filter bank is used, which is based on the 1-D example above. We apply the separable filter bank on the $512 \times 512$ picture "Lenna." The original image with 8 b/pixel is shown in Fig. 8(a). Bit allocation and entropy coding are performed in the subbands. The reconstructed image shown in Fig. 8(b) has a subband bit rate of $0.35 \mathrm{~b}$ /pixel. The peak signal-to-noise ratio (PSNR) is $35.5 \mathrm{~dB}$.

$$
\begin{aligned}
& \text { PSNR } \triangleq \\
& 10 \log _{10} \frac{\text { (peak-to-peak value of the original image) })^{2}}{\text { MSE }}
\end{aligned}
$$

where MSE is the mean square error of the reconstructed image. We notice that the reconstruction quality is very good and shows no artifacts due to the excess passband width discussed at the beginning of Section V.

Example 6.3-PR System with $M=19$, i.e., 38 Channels: The prototype $P_{0}(z)$ in this example is of order 133. Following the discussion in Section III, the number of nonzero samples of $P_{0}(z)$ is 110 . It has stopband attenuation $40 \mathrm{~dB}$ and stopband edge $\omega_{s} 0.06 \pi$. Fig. 9(a) and (b) show, respectively, normalized magnitude responses of the first set of analysis filters and the second set of analysis filters.

In both of these examples, the analysis filters have linear phase by construction; therefore, we have not shown the phase responses. In the second example, the system has PR property by construction; therefore, we have not shown any aliasing error or the magnitude response of the distortion function.

Tables of Prototype Filter Coefficients for Perfect Reconstruction: We list two groups of filter banks. Only the coefficients of the prototype filters $p_{0}(n)$ are listed. The filters in the first group are of order $N=3 M$ (where the meaning of $M$ is the same as in Fig. 2). The filters in the second group has order $N=7 M$. The prototype filters are linear-phase; only the first half of the coefficients are shown. From the coefficients of the prototype $P_{0}(z)$ we can find the coefficients of all analysis and synthesis filters using (12).

1) Filters with order $3 M$ : Prototype filter coefficients $p_{0}(n)$ are listed in Table I. Filters in this group have stop band attenuation $A_{s} \approx 25 \mathrm{~dB}$.

2) Filters with order $7 M$ : Prototype filter coefficients $p_{0}(n)$ are listed in Table II. Filters in this group have $A_{s} \approx 40$ $\mathrm{dB}$.

Notice that in Tables I and II every prototype filter starts with $\left\lceil\frac{M+1}{2}\right\rceil$ zeros. The notation \lceil\rceil and the notation \lfloor\rfloor 
TABLE II

Prototype Filters with Stopband Attenuation About $40 \mathrm{~dB}$ and Order $7 \mathrm{M}$ : Part I

n $\quad M=7 \quad M=9 \quad M=11 \quad M=13 \quad M=15 \quad M=17 \quad$

\begin{tabular}{|c|c|c|c|c|c|c|}
\hline 0 & $0.0000000 \mathrm{E}+00$ & $0.0000000 \mathrm{E}+00$ & $0.0000000 \mathrm{E}+00$ & $0.0000000 \mathrm{E}+00$ & $0.0000000 \mathrm{E}+00$ & $0.0000000 \mathrm{E}+00$ \\
\hline t & $0.0000000 \mathrm{E}+00$ & $0.0000000 \mathrm{E}+00$ & $0.0000000 \mathrm{E}+00$ & $0.0000000 \mathrm{E}+00$ & $0.0000000 \mathrm{E}+00$ & $0.0000000 E+00$ \\
\hline 2 & $0.0000000 \mathrm{E}+00$ & $0.0000000 \mathrm{E}+00$ & $0.0000000 \mathrm{E}+00$ & $0.0000000 \mathrm{E}+00$ & $0.0000000 \mathrm{E}+00$ & $0.0000000 \mathrm{E}+00$ \\
\hline & $0.0000000 \mathrm{E}+00$ & $0.0000000 \mathrm{E}+00$ & $0.0000000 \mathrm{E}+00$ & $0.0000000 \mathrm{E}+00$ & $0.0000000 \mathrm{E}+00$ & $0.0000000 \mathrm{E}+00$ \\
\hline 4 & $-6.7978138 \mathrm{E}-04$ & $0.0000000 \mathrm{E}+00$ & $0.0000000 \mathrm{E}+00$ & $0.0000000 \mathrm{E}+00$ & $0.0000000 \mathrm{E}+00$ & $0.0000000 \mathrm{E}+00$ \\
\hline 5 & $-6.7628801 \mathrm{E}-04$ & $-5.1424337 \mathrm{E}-04$ & $0.0000000 \mathrm{E}+00$ & $0.0000000 \mathrm{E}+00$ & $0.0000000 \mathrm{E}+00$ & $0.0000000 \mathrm{E}+00$ \\
\hline & $-4.0454551 \mathrm{E}-04$ & $-5.5565799 \mathrm{E}-04$ & $-4.1089306 \mathrm{E}-04$ & $0.0000000 \mathrm{E}+00$ & $0.0000000 \mathrm{E}+00$ & $0.0000000 \mathrm{E}+00$ \\
\hline 7 & $0.0000000 \mathrm{E}+00$ & $-4.6574969 \mathrm{E}-04$ & $-4.5550830 \mathrm{E}-04$ & $-2.3753750 \mathrm{E}-04$ & $0.0000000 \mathrm{E}+00$ & $0.0000000 \mathrm{E}+00$ \\
\hline 8 & $-7.6992401 \mathrm{E}-04$ & $-2.8685604 \mathrm{E}-04$ & $-4.3325456 \mathrm{E}-04$ & $-2.6818115 \mathrm{E}-04$ & $-3.3451450 \mathrm{E}-04$ & $0.0000000 \mathrm{E}+00$ \\
\hline 9 & $-2.0355803 \mathrm{E}-03$ & $0.0000000 \mathrm{E}+00$ & $-3.4597558 \mathrm{E}-04$ & $-2.6459598 \mathrm{E}-04$ & $-3.6708079 \mathrm{E}-04$ & $-2.8913044 \mathrm{E}-04$ \\
\hline 10 & $-3.4812475 \mathrm{E}-03$ & $-5.3310500 \mathrm{E}-04$ & $-2.2815828 \mathrm{E}-04$ & $-2.1799966 \mathrm{E}-04$ & $-3.7170523 E-04$ & $-3.1707660 \mathrm{E}-04$ \\
\hline 11 . & $-4.3485166 \mathrm{E}-03$ & $-1.1604805 E-03$ & $0.0000000 \mathrm{E}+00$ & $-1.3245846 \mathrm{E}-04$ & $-3.4512883 E-04$ & $-3.2738333 \mathrm{E}-04$ \\
\hline 12 & $-4.1820964 \mathrm{E}-03$ & $-2.0107373 \mathrm{E}-03$ & $-4.4237113 \mathrm{E}-04$ & $8.4582874 \mathrm{E}-05$ & $-2.9116705 \mathrm{E}-04$ & $-3.1725513 \mathrm{E}-04$ \\
\hline 13 & $-2.7657296 \mathrm{E}-03$ & $-2.8242451 \mathrm{E}-03$ & $-7.8913118 \mathrm{E}-04$ & $0.0000000 \mathrm{E}+00$ & $-2.2215026 \mathrm{E}-04$ & $-2.8713764 \mathrm{E}-04$ \\
\hline 14 & $0.0000000 \mathrm{E}+00$ & $-3.3206044 \mathrm{E}-03$ & $-1.2985038 \mathrm{E}-03$ & $-6.0356928 \mathrm{E}-05$ & $-1.5212153 \mathrm{E}-04$ & $-2.4166190 \mathrm{E}-04$ \\
\hline 15 & $5.2636888 \mathrm{E}-03$ & $-3.3414132 \mathrm{E}-03$ & $-1.8618945 \mathrm{E}-03$ & $-2.4826199 \mathrm{E}-04$ & $0.0000000 \mathrm{E}+00$ & $-1.8912721 \mathrm{E}-04$ \\
\hline 16 & $1.2587822 \mathrm{E}-02$ & $-2.8254978 \mathrm{E}-03$ & $-2.3635259 \mathrm{E}-03$ & $-5.6044074 \mathrm{E}-04$ & $-2.8745428 \mathrm{E}-04$ & $-1.3245575 \mathrm{E}-04$ \\
\hline 17 & $2.2269310 \mathrm{E}-02$ & $-1.6758764 \mathrm{E}-03$ & $-2.6749144 \mathrm{E}-03$ & $-9.2668924 \mathrm{E}-04$ & $-4.3298166 \mathrm{E}-04$ & $0.0000000 \mathrm{E}+00$ \\
\hline 18 & $3.3964824 \mathrm{E}-02$ & $0.0000000 \mathrm{E}+00$ & $-2.7285117 \mathrm{E}-03$ & $-1.2837077 \mathrm{E}-03$ & $-6.6823280 \mathrm{E}-04$ & $-2.7298008 \mathrm{E}-04$ \\
\hline 19 & $4.6897783 \mathrm{E}-02$ & $3.1145173 \mathrm{E}-03$ & $-2.5059040 \mathrm{E}-03$ & $-1.5795348 \mathrm{E}-03$ & $-9.6850692 \mathrm{E}-04$ & $-3.7133376 \mathrm{E}-04$ \\
\hline 20 & $6.0046992 \mathrm{E}-02$ & $7.0401227 \mathrm{E}-03$ & $-1.9701690 \mathrm{E}-03$ & $-1.7616044 \mathrm{E}-03$ & $-1.3016585 \mathrm{E}-03$ & $-5.3290996 \mathrm{E}-04$ \\
\hline 21 & $7.2291059 \mathrm{E}-02$ & $1.2091438 \mathrm{E}-02$ & $-1.0762419 \mathrm{E}-03$ & $-1.8101016 \mathrm{E}-03$ & $-1.6320878 \mathrm{E}-03$ & $-7.4432310 \mathrm{E}-04$ \\
\hline 22 & $8.2524184 \mathrm{E}-02$ & $1.8236891 \mathrm{E}-02$ & $0.0000000 \mathrm{E}+00$ & $-1.7319940 \mathrm{E}-03$ & $-1.9243028 \mathrm{E}-03$ & $-9.8761395 \mathrm{E}-04$ \\
\hline 23 & $8.9844578 \mathrm{E}-02$ & $2.5315655 \mathrm{E}-02$ & $2.0867021 \mathrm{E}-03$ & $-1.5293427 \mathrm{E}-03$ & $-2.1297547 \mathrm{E}-03$ & $-1.2421812 \mathrm{E}-03$ \\
\hline 24 & $9.3653468 \mathrm{E}-02$ & $3.3037717 \mathrm{E}-02$ & $4.4937327 \mathrm{E}-03$ & $-1.1957418 \mathrm{E}-03$ & $-2.2244700 \mathrm{E}-03$ & $-1.4866742 \mathrm{E}-03$ \\
\hline 25 & & $4.1034799 \mathrm{E}-02$ & $7.5104250 \mathrm{E}-03$ & $-6.1052640 \mathrm{E}-06$ & $-2.2049230 \mathrm{E}-03$ & $-1.7003521 \mathrm{E}-03$ \\
\hline 26 & & $4.8905760 \mathrm{E}-02$ & $1.1152817 \mathrm{E}-02$ & $0.0000000 \mathrm{E}+00$ & $-2.0540988 \mathrm{E}-03$ & $-1.8521292 \mathrm{E}-03$ \\
\hline 27 & & $5.6230719 \mathrm{E}-02$ & $1.5386557 \mathrm{E}-02$ & $-4.3566146 \mathrm{E}-06$ & $-1.7561930 \mathrm{E}-03$ & $-1.9280654 \mathrm{E}-03$ \\
\hline 28 & & $6.2603026 \mathrm{E}-02$ & $2.0128782 \mathrm{E}-02$ & $2.2411346 \mathrm{E}-03$ & $-1.2950668 \mathrm{E}-03$ & $-1.9291234 \mathrm{E}-03$ \\
\hline 29 & & $6.7681773 \mathrm{E}-02$ & $2.5246480 \mathrm{E}-02$ & $3.9316848 \mathrm{E}-03$ & $-6.5704476 \mathrm{E}-04$ & $-1.8450335 \mathrm{E}-03$ \\
\hline 30 & & $7.1207984 \mathrm{E}-02$ & $3.0574428 \mathrm{E}-02$ & $6.0659284 \mathrm{E}-03$ & $0.0000000 E+00$ & $-1.6665926 E-03$ \\
\hline 31 & & $7.3014065 \mathrm{E}-02$ & $3.5936126 \mathrm{E}-02$ & $8.6644470 \mathrm{E}-03$ & $1.2415753 \mathrm{E}-03$ & $-1.3847337 \mathrm{E}-03$ \\
\hline 32 & & & $4.1144825 \mathrm{E}-02$ & $1.1714005 \mathrm{E}-02$ & $2.5241480 \mathrm{E}-03$ & $\begin{array}{l}-1.5845707 \mathrm{E}-04 \\
-9.89847\end{array}$ \\
\hline 33 & & & $4.6012297 \mathrm{E}-02$ & 1.5171981E-02 & $4.0304895 \mathrm{E}-03$ & $-4.7750042 \mathrm{E}-04$ \\
\hline 34 & & & $5.0354801 \mathrm{E}-02$ & $1.8965488 \mathrm{E}-02$ & $5.7642502 \mathrm{E}-03$ & 0.00000 \\
\hline 35 & & & $5.4018886 \mathrm{E}-02$ & $2.3001951 \mathrm{E}-02$ & $7.7213246 \mathrm{E}-03$ & 9.84087 \\
\hline 36 & & & $5.6876247 \mathrm{E}-02$ & $2.7175943 \mathrm{E}-02$ & $9.8902761 \mathrm{E}-03$ & $1.9434730 \mathrm{E}-03$ \\
\hline 37 & & & $5.8833442 \mathrm{E}-02$ & $3.1369115 \mathrm{E}-02$ & $1.2251466 \mathrm{E}-02$ & $3.0535983 \mathrm{E}-03$ \\
\hline 38 & & & $5.9828410 \mathrm{E}-02$ & $3.5456422 \mathrm{E}-02$ & $1.4779999 \mathrm{E}-02$ & 4.3201697E-03 \\
\hline 39 & & & & $3.9299808 \mathrm{E}-02$ & $1.7440394 \mathrm{E}-02$ & $5.7435820 \mathrm{E}-03$ \\
\hline 40 & & & & $4.2799312 \mathrm{E}-02$ & $2.0188871 \mathrm{E}-02$ & $7.3196175 \mathrm{E}-03$ \\
\hline 41 & & & & $4.5808262 \mathrm{E}-02$ & $2.2982202 \mathrm{E}-02$ & $9.0401028 \mathrm{E}-03$ \\
\hline 42 & & & & $4.8293471 \mathrm{E}-02$ & $2.5774117 \mathrm{E}-02$ & $1.0892218 \mathrm{E}-02$ \\
\hline 43 & & & & $5.0190996 \mathrm{E}-02$ & $2.8515837 \mathrm{E}-02$ & $1.2860679 \mathrm{E}-02$ \\
\hline 44 & & & & $5.1470294 \mathrm{E}-02$ & $3.1157139 \mathrm{E}-02$ & $1.4923902 \mathrm{E}-02$ \\
\hline 45 & & & & $5.2114185 \mathrm{E}-02$ & $3.3648561 \mathrm{E}-02$ & $1.7055081 \mathrm{E}-02$ \\
\hline 46 & & & & & $35938906 \mathrm{E}-02$ & $1.9227724 \mathrm{E}-02$ \\
\hline 47 & & & & & $3.7987010 \mathrm{E}-02$ & $2.1414194 \mathrm{E}-02$ \\
\hline 48 & & & & & $3.9752809 \mathrm{E}-02$ & $2.3584936 \mathrm{E}-02$ \\
\hline 49 & & & & & $4.1203964 \mathrm{E}-02$ & $2.5709521 \mathrm{E}-02$ \\
\hline 50 & & & & & $4.2314864 \mathrm{E}-02$ & $2.7756975 \mathrm{E}-02$ \\
\hline 51 & & & & & $4.3066456 \mathrm{E}-02$ & $2.9697395 \mathrm{E}-02$ \\
\hline 52 & & & & & 4.3445987E-02 & $3.1498132 \mathrm{E}-02$ \\
\hline 53 & & & & & & 3.3135487E-02 \\
\hline 54 & & & & & & $3.4583767 \mathrm{E}-02$ \\
\hline 55 & & & & & & $3.5822129 \mathrm{E}-02$ \\
\hline 56 & & & & & & $3.6833357 \mathrm{E}-02$ \\
\hline 57 & & & & & & $3.7603806 \mathrm{E}-02$ \\
\hline 58 & & & & & & $3.8123431 \mathrm{E}-02$ \\
\hline 59 & & & & & & $3.8385256 \mathrm{E}-02$ \\
\hline
\end{tabular}

to be used later are defined in Section I. Those zeros at the beginning of the prototype filters are the result of optimization. As indicated in Section III, $g_{k}(0)$ or $g_{k}\left(m_{0}\right)$ must be zero for $k=1,2, \ldots, M-1$, where $g_{k}(n)$ is the $k$ th polyphase component of $p_{0}(n)$. After optimization, we found that it is best to choose

$$
\begin{aligned}
& g_{0}(n)=\delta\left(n-\left\lfloor\frac{m_{0}+1}{2}\right\rfloor\right) \\
& g_{k}(0)=0, \quad k=1,2, \ldots,\left\lfloor\frac{M-1}{2}\right\rfloor
\end{aligned}
$$

where the unit impulse function $\delta(n)$ is defined in Section I.
By linear-phase constraint of $P_{0}(z)$, we have

$$
\begin{aligned}
& g_{M}(n)=\delta\left(n-\left\lceil\frac{m_{0}-1}{2}\right\rceil\right), \\
& g_{k}\left(m_{0}\right)=0, \quad k=\left\lceil\frac{M+1}{2}\right\rceil, \ldots, M-1 .
\end{aligned}
$$

Those zeros at the beginning of the prototype filters can not be removed without shifting the cosine modulation in (12). To be consistent with (12), we keep the zeros in the tables. If the quantity $p_{0}(n)$ is directly used in (12) with no modifications, then the set of analysis and synthesis filters have linear phase and give perfect reconstruction. 
TABLE II

Prototype Fliters with Stopband Attenuation About 40 dB and ORder $7 M$ : Part II

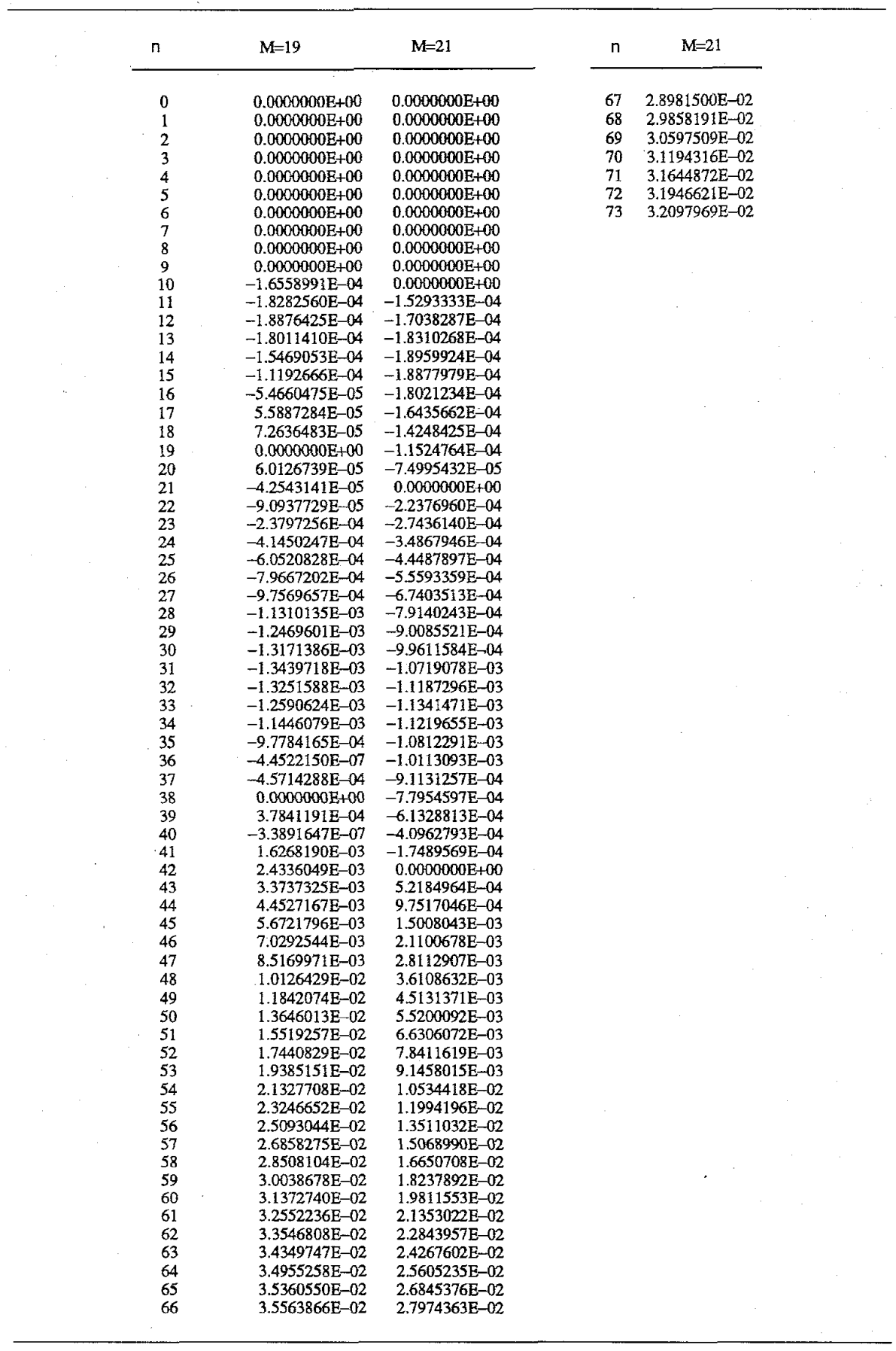

\section{CONCLUDING REMARKS}

In this paper, we introduced a new class of maximally decimated cosine modulated systems, which have the following properties:

1) Aliasing is canceled exactly or approximately as desired.

2) Amplitude distortion function can be designed to be flat by optimizing over prototype filter coefficients.

3) Each analysis and synthesis filter in the filter bank has linear phase.
4) This new $2 M$ channel maximally decimated cosine modulated filter bank has the same design cost and implementation cost as conventional $M$ channel maximally decimated cosine modulated filter banks. Correspondingly, the coding gain is nearly identical to that of an $M$ channel paraunitary system.

5) Perfect reconstruction is possible if polyphase components of the prototype filter satisfy the two conditions given in Section III. Linear phase property of each analysis and synthesis filter is still preserved in this case. 


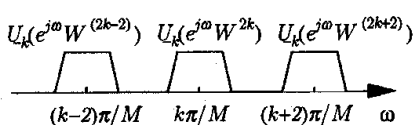

(a)

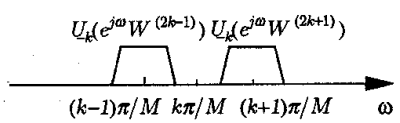

(b)

Fig. 10. Image copies of $U_{-k}(z)$ due to decimation followed by expansion.

Summarizing, we have developed a linear phase cosine modulated maximally decimated perfect reconstruction system. Design examples show that very good attenuation characteristics can be obtained as well.

\section{APPENDIX A}

The quantity $A_{i}^{(1)}(z)$ in (9) is zero if $1+W^{-M i}=0$ or $U_{k}\left(z W^{i}\right) \tilde{U}_{k}(z)=0$ for $k=1,2, \ldots, M-1$. Since $W^{-M i}=-1$ for odd $i$ and by $(5) U_{k}\left(z W^{i}\right) \tilde{U}_{k}(z) \approx 0$, for $i \neq 1, A_{i}^{(1)}(z)$ in (9) is approximately 0 . So is $A_{i}^{(4)}(z)$ for the same reason.

Similarly $A_{i}^{(2)}(z)$ is zero if $a_{k}^{* 2}+a_{k}^{\prime * 2} W^{-M i}=0$ or $U_{-k}\left(z W^{i}\right) \tilde{U}_{k}(z) \approx 0$, for $k=1,2, \ldots, M-1$. The case when $i$ is even and the case when $i$ is odd are discussed separately.

1) $i$ is even: From Fig. 10(a), we observe that only the term $k=i / 2$ is the major nonzero part of $A_{i}(z)$. By choosing $a_{k}=1$ and $a_{k}^{\prime}=-\sqrt{-1}, k=1, \ldots, M-1$, it can be completely eliminated.

2) $i$ is odd: Consider two cases:

a) $1<i<2 M-1$ : By using (5) and (9), we have

$$
\begin{aligned}
A_{i}^{(2)}(z) \approx & 2 U_{-\frac{i-1}{2}}\left(z W^{i}\right) \tilde{U}_{\frac{i-1}{2}}(z) \\
& +2 U_{-\frac{i+1}{2}}\left(z W^{i}\right) \tilde{U}_{\frac{i+1}{2}}(z) .
\end{aligned}
$$

We can also observe the result in (A.1) from Fig. 10(b). Since $P_{0}(z)$ has linear phase, $P_{0}\left(e^{j \omega}\right)=e^{-j \omega N / 2} P_{R}(\omega)$, where $P_{R}(\omega)$ is a real-valued function. We can rewrite (A.1) in terms of $P_{R}(\omega)$ as

$$
\begin{aligned}
A_{i}^{(2)}(z) \approx & 2\left(W^{N / 2}+W^{-N / 2}\right) P_{R}\left(\omega-\frac{\pi}{M} \frac{i-1}{2}\right) \\
& P_{R}\left(\omega-\frac{\pi}{M} \frac{i+1}{2}\right) .
\end{aligned}
$$

If $N=\left(2 m_{0}+1\right) M$ for some positive integer $m_{0}$, then $\left(W^{N / 2}+W^{-N / 2}\right)=0$. In this case $A_{i}^{(2)}(z)$ in (9) is approximately zero.

b) $i=1$ or $2 M-1$ : When $i=1, A_{1}^{(2)}(z)$ has a major nonzero term, $2 U_{-1}(z W) \tilde{U}_{1}(z)$, which cancels the term $2 U_{0}(z W) \tilde{U}_{0}(z)$ in (9) with the same choice of $N$. This is similar for $i=2 M-1$.

The cancellation of $A_{i}^{(3)}(z)$ is similar to the cancellation of the $A_{i}^{(2)}(z)$.

\section{APPENDIX B \\ PROOF OF (23)}

To derive (23), we need to prove the following properties of $\mathbf{C}$ and $\mathbf{S}$ :

$$
\begin{aligned}
& \mathbf{C}^{T} \mathbf{C}=\frac{M}{2}\left(\begin{array}{cc}
2 & 0 \\
\mathbf{0} & \mathbf{I}_{M-1}
\end{array}\right) \\
& \mathbf{C}^{T} \boldsymbol{\Lambda}_{1} \mathbf{C}=\frac{M}{2}\left(\begin{array}{cc}
0 & \mathbf{0} \\
\mathbf{0} & \mathbf{J}_{M-1}
\end{array}\right) \\
& \mathbf{S}^{T} \mathbf{S}=\frac{M}{2}\left(\begin{array}{cc}
0 & 0 \\
0 & \mathbf{I}_{M-1}
\end{array}\right) \\
& \mathbf{S}^{T} \boldsymbol{\Lambda}_{2} \mathbf{S}=\frac{M}{2}\left(\begin{array}{cc}
0 & 0 \\
0 & \mathbf{J}_{M-1}
\end{array}\right)
\end{aligned}
$$

If (B.1.a)-(B.1.d) are true, then (23) follows.

Matrix $\mathbf{C}$ is very similar to a $(M+1) \times(M+1)$ type I DCT matrix, $\mathbf{C}_{M+1}^{I}$, and $\mathbf{S}$ is very similar to a $(M-1) \times(M-1)$ type I DST matrix, $\mathbf{S}_{M-1}^{I}$. (Both $\mathbf{C}_{M+1}^{I}$ and $\mathbf{S}_{M-1}^{I}$ are documented in [21].) $\mathbf{C}_{M+1}^{I}$ and $\mathbf{S}_{M-1}^{I}$ have entries

$$
\begin{aligned}
{\left[\mathbf{C}_{M+1}^{I}\right]_{m n}=\kappa_{m} \kappa_{n} \sqrt{\frac{2}{M}} \cos \left(\frac{\pi}{M} m n\right), } & 0 \leq m, n \leq M \\
{\left[\mathbf{S}_{M-1}^{I}\right]_{m n}=\sqrt{\frac{2}{M}} \sin \left(\frac{\pi}{M} m n\right), } & 1 \leq m, n \leq M-1 .
\end{aligned}
$$

Several useful properties of $\mathbf{C}_{M+1}^{I}$ and $\mathbf{S}_{M-1}^{I}$ are stated in the following to help proving (B.1).

$$
\begin{aligned}
& \mathbf{C}_{M+1}^{I}{ }^{T} \mathbf{C}_{M+1}^{I}=\mathbf{I}_{M+1} \\
& \mathbf{C}_{M+1}^{I}{ }^{T} \Lambda_{1} \mathbf{C}_{M+1}^{I}=\mathbf{J}_{M+1} \\
& \mathbf{S}_{M-1}^{I}{ }^{T} \mathbf{S}_{M-1}^{I}=\mathbf{I}_{M-1}, \\
& \mathbf{S}_{M-1}^{I}{ }^{T} \Lambda_{2} \mathbf{S}_{M-1}^{I}=-\mathbf{J}_{M-1}
\end{aligned}
$$

Proof of (B.1.a)-(B.I.d): $\mathbf{C}$ and $\mathbf{S}$ are related to $\mathbf{C}_{M+1}^{I}$ and $\mathbf{S}_{M-1}^{I}$ as

$$
\sqrt{\frac{M}{2}} \mathbf{C}_{M+1}^{I}=\left(\begin{array}{ll}
\mathbf{C} \boldsymbol{\Gamma} & \mathbf{r}
\end{array}\right), \quad \mathbf{S}=\left(\begin{array}{ll}
\mathbf{0} & \sqrt{\frac{M}{2}} \mathbf{S}_{M-1}^{I}
\end{array}\right)
$$

where $\mathbf{r}$ is the last column of $\sqrt{\frac{M}{2}} \mathbf{C}_{M+1}^{I}$ and $\boldsymbol{\Gamma}=$ $\left(\begin{array}{cc}\sqrt{\frac{1}{2}} & \mathbf{0} \\ \mathbf{0} & \mathbf{I}_{M-1}\end{array}\right)$ with inverse $\boldsymbol{\Gamma}^{-1}=\left(\begin{array}{cc}\sqrt{2} & \mathbf{0} \\ \mathbf{0} & \mathbf{I}_{M-1}\end{array}\right)$. By (B.2.a), we have

$$
\frac{M}{2} \mathbf{C}_{M+1}^{I}{ }^{T} \mathbf{C}_{M+1}^{I}=\frac{M}{2} \mathbf{I}_{M+1} .
$$

and from (B.3), we have

$$
\frac{M}{2} \mathbf{C}_{M+1}^{I}{ }^{T} \mathbf{C}_{M+1}^{I}=\left(\begin{array}{cc}
\Gamma^{T} \mathbf{C}^{T} \mathbf{C r} & \boldsymbol{\Gamma}^{T} \mathbf{C}^{T} \mathbf{r} \\
\mathbf{r}^{T} \mathbf{C} \boldsymbol{\Gamma} & \mathbf{r}^{T} \mathbf{r}
\end{array}\right)
$$

Comparing (B.4) and (B.5) gives us

$$
\boldsymbol{\Gamma}^{T} \mathbf{C}^{T} \mathbf{C} \boldsymbol{\Gamma}=\frac{M}{2} \mathbf{I}_{M}
$$

Relation (B.1.a) follows the above equation. Similarly for (B.1.c). Relations (B.1.b) and (B.1.d) can be proved in a similar manner. 


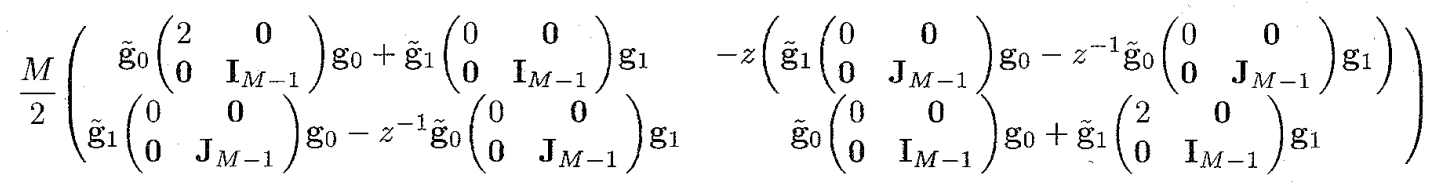

With (B.1), the right-hand side of (22) can be written as the matrix at the top of this page. The dependence of $\mathrm{g}_{i}$ on $z$ is dropped for convenience. Notice that the off diagonal elements in the above big matrix is 0 if and only if

$$
\begin{gathered}
\tilde{G}_{k+M}(z) G_{M-k}(z)-z^{-1} \tilde{G}_{k}(z) G_{2 M-k}(z)=0 \\
k=1, \ldots, M-1
\end{gathered}
$$

Because the prototype filter $P_{0}(z)$ is of order $N=\left(2 m_{0}+\right.$ 1) $M$ and has linear phase, $G_{k}(z)$ is related to $G_{M-k}(z)$ and $G_{k+M}(z)$ is related to $G_{2 M-k}(z)$, for $k=1,2, \ldots, M-1$.

$$
\begin{aligned}
G_{k}(z) & =z^{-m_{0}} \tilde{G}_{M-k}(z), \\
G_{k+M}(z) & =z^{-\left(m_{0}-1\right)} \tilde{G}_{2 M-k}(z) \\
& k=1,2, \ldots, M-1 .
\end{aligned}
$$

The above property makes (B.6) automatically satisfied.

\section{APPENDIX C}

From the definitions of $\mathrm{T}_{1}$ and $\mathrm{T}_{2}$, we know

$$
\mathbf{d}_{1}=\mathrm{Ca}_{1}+\mathbf{\Lambda}_{1} \mathbf{C a}_{2}, \quad \text { and } \quad \mathbf{d}_{2}=\mathbf{S b}_{1}+\boldsymbol{\Lambda}_{2} \mathbf{S b}_{2} .
$$

Using the relations of (B.3) and (B.1), we can write

$$
\Lambda_{1}\left(\begin{array}{ll}
C \Gamma & \mathbf{r}
\end{array}\right)=\left(\begin{array}{ll}
C \Gamma & \mathbf{r}
\end{array}\right) \mathbf{J}_{M+1}
$$

and

$$
\Lambda_{2}\left(\begin{array}{ll}
\mathbf{0} & \mathbf{S}_{M-1}^{I}
\end{array}\right)=-\mathbf{S}_{M-1}^{I}\left(\mathbf{0} \cdot \mathbf{J}_{M-1}\right)
$$

That gives us

$$
\boldsymbol{\Lambda}_{1} \mathbf{C}=\mathbf{C}\left(\begin{array}{cc}
0 & 0 \\
0 & \mathbf{J}_{M-1}
\end{array}\right)+\sqrt{2}\left(\begin{array}{ll}
\mathbf{r} & 0
\end{array}\right)
$$

and

$$
\Lambda_{2} \mathbf{S}=-\mathbf{S}\left(\begin{array}{cc}
0 & 0 \\
0 & \mathbf{J}_{M-1}
\end{array}\right) .
$$

Substituting (C.2) into (C.1), we obtain (26).

\section{ACKNOWLEDGMENT}

The authors would like to thank S.-M. Phoong and I. Djokovic for fruitful discussion.

\section{REFERENCES}

[1] R. E. Crochiere and L. R. Rabiner, Multirate Digital Signal Processing. Englewood Cliffs, NJ: Prentice Hall, 1983.

[2] M. J. T. Smith and T. P. Barnwell, "A new filter bank theory for time-frequency representation," IEEE Trans. Acoust., Speech Signal Processing, vol. ASSP-35, pp. 314-327, Mar. 1987.

[3] M. A. Vetterli, "A theory of multirate filter banks," IEEE Trans. Acoust., Speech Signal Processing, vol. ASSP-35, pp. 356-372, Mar. 1987.
[4] P. P. Vaidyanathan, "Quadrature mirror filter banks, $M$-band extensions and perfect reconstruction techniques," IEEE Acoust., Speech Signal Processing Mag., vol. 4, pp. 4-20, June 1987.

[5] H. J. Nussbaumer, "Pseudo QMF filter bank," IBM Tech. Disclosure Bull., vol. 24, pp. 3081-3087, Nov. 1981

[6] J. H. Rothweiler, "Polyphase quadrature filters, a new subband coding technique," in Proc. IEEE Int. Conf. Acoust., Speech Signal Processing, Apr. 1983 , pp. 1980-1983.

[7] P. L. Chu, "Quadrature mirror filter design for an arbitrary number of equal bandwidth channels," IEEE Trans. Acoust., Speech Signal Processing, vol. ASSP-33, pp. 203-218, Feb. 1985.

[8] J. Masson and Z. Picel, "Flexible design of computationally efficient nearly perfect QMF filter banks," in Proc. IEEE Int. Conf. Acoust., Speech Signal Processing, Tampa, FL, Mar. 1985, pp. 14.7.1-14.7.4.

[9] R. V. Cox, "The design of uniformly and nonuniformly spaced pseudo QMF," IEEE Trans. Acoust., Speech Signal Processing, vol. ASSP-34, pp. 1090-1096, Oct. 1986

[10] J. P. Princen and A. B. Bradley, "Analysis/synthesis filter bank design based on time domain aliasing cancellation," IEEE Trans. Acoust., Speech Signal Processing, vol. ASSP-34, pp. 1153-1161, 1986.

[11] R. D. Koilpillai and P. P. Vaidyanathan, "Cosine-modulated FIR filter banks satisfying perfect reconstruction," IEEE Trans. Signal Processing, vol. 40, pp. $770-783$, Apr. 1992.

[12] T. A. Ramstad, "Cosine modulated analysis-synthesis filter banks with critical sampling and perfect reconstruction," in Proc. IEEE Int. Conf. Acoust., Speech Signal Processing, Toronto, Canada, May 1991, pp. 1789-1792.

[13] H. S. Malvar, Signal Processing With Lapped Transforms. Norwood, MA: Artech House, 1992.

[14] T. Q. Nguyen, "A class of generalized cosine-modulated filter bank," in Proc. IEEE Int. Symp. Circuits Syst., 1992.

[15] R. A. Gopinath and C. S. Burrus, "Theory of modulated filter banks and modulated tight frames," in Proc. IEEE Int. Conf. Acoust., Speech Signal Processing, 1993.

[16] A. K. Soman, P. P. Vaidyanathan, and T. Q. Nguyen, "Linear phase paraunitary filter banks: theory, factorizations and applications," Tech. Rep., Calif. Inst. of Technol., Pasadena, CA, May 1992.

[17] P. P. Vaidyanathan, Multirate Systems and Filter Banks. Englewood Cliffs, NJ: Prentice Hall, 1993.

[18] N. J. Fliege, "Computational efficiency of modified DFT polyphase filter banks," in Proc. 27th Ann. Asilomar Conf. Signals, Syst. Comput., 1993.

[19] P. P. Vaidyanathan, "Orthonormal and biorthonormal filter banks as convolvers, and convolutional coding gain," IEEE Trans. Signal Processing, vol. 41, pp. 2110-2130, June 1993.

[20] W. H. Press, B. P. Flannery, S. A. Teukolsky, and W. T. Vetterling, Numerical Recipes. Cambridge, UK: Cambridge Univ. Press, 1989.

[21] P. Yip and K. R. Rao, "Fast discrete transforms," in Handbook of Digital Signal Processing, D. F. Elliott, Ed. San Diego, CA: Academic, 1987.

[22] A. Papoulis, Signal Analysis. New York: McGraw-Hill, 1965.

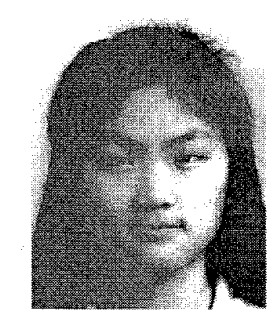

Yuan-Pei Lin (S'93) was born in Taipei, Taiwan, in 1970. She received the B.S. degree in control engineering from the National Chiao-Tong University, Hsinchu, Taiwan, in 1992 and the M.S. degree in electrical engineering from the California Institute of Technology, Pasadena, in 1993. She is currently pursuing the doctoral degree at the California Institute of Technology.

Her interests include digital signal processing and multidimensional filter banks. 


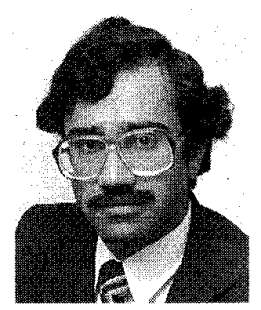

P. P. Vaidyanathan (S'80-M'83-SM'88-F'91) was born in Calcutta, India, on October 16, 1954. He received the B.Sc. (honors) degree in physics and the B.Tech. and M.Tech. degrees in radiophysics and electronics, all from the University of Calcutta, India, in 1974, 1977, and 1979, respectively, and the $\mathrm{Ph} . \mathrm{D}$. degree in electrical and computer engineering from the University of California at Santa Barbara in 1982. He was a postdoctoral fellow at the University of California, Santa Barbara, during the 1982-1983 academic year.

In 1983, he joined the electrical engineering department of the California Institute of Technology, Pasadena, as an Assistant Professor and has been Professor since 1993. His main research interests are in digital signal processing, multirate systems, wavelet transforms, and adaptive filtering. He was an Associate Editor for IEEE TRANSACTIONS ON CIRCUITS AND SySTEMS from 1985 to 1987 and is currently an Associate Editor for IEEE SIGNAL PROCESSING LETTERS and a Consulting Editor for Applied and Computational Harmonic Analysis. He has authored a number of papers in IEEE journals and is the author of the book Multirate Systems and Filter Banks. He has written several chapters for various signal processing handbooks.

Dr. Vaidyanathan served as Vice Chairman of the Technical Program committee for the 1983 IEEE International Symposium on Circuits and Systems, and as the Technical Program Chairman for the 1992 IEEE International Symposium on Circuits and Systems. He was a recipient of the Award for excellence in teaching at the California Institute of Technology for the years 1983-1984, 1992-1993, and 1993-1994. He also received the NSF's Presidential Young Investigator award in 1986. In 1989, he received the IEEE ASSP Senior Award for his paper on multirate perfect-reconstruction filter banks. In 1990, he was recipient of the S. K. Mitra Memorial Award from the Institute of Electronics and Telecommunications Engineers, India, for his joint paper in the IETE Journal. He is the 1995 recipient of the F. E. Terman award from the ASEE, which is sponsored by Hewlett-Packard. 